\title{
Metabolic engineering of microorganisms for production of aromatic compounds
}

\author{
Damla Huccetogullari ${ }^{1,2}$, Zi Wei Luo $0^{1,2}$ and Sang Yup Lee ${ }^{1,2,3^{*}}$ (D)
}

\begin{abstract}
Metabolic engineering has been enabling development of high performance microbial strains for the efficient production of natural and non-natural compounds from renewable non-food biomass. Even though microbial production of various chemicals has successfully been conducted and commercialized, there are still numerous chemicals and materials that await their efficient bio-based production. Aromatic chemicals, which are typically derived from benzene, toluene and xylene in petroleum industry, have been used in large amounts in various industries. Over the last three decades, many metabolically engineered microorganisms have been developed for the bio-based production of aromatic chemicals, many of which are derived from aromatic amino acid pathways. This review highlights the latest metabolic engineering strategies and tools applied to the biosynthesis of aromatic chemicals, many derived from shikimate and aromatic amino acids, including L-phenylalanine, L-tyrosine and L-tryptophan. It is expected that more and more engineered microorganisms capable of efficiently producing aromatic chemicals will be developed toward their industrial-scale production from renewable biomass.
\end{abstract}

Keywords: Aromatic compounds, Metabolic engineering, Synthetic biology, Shikimate pathway, Phenylalanine, Tyrosine, Tryptophan

\section{Background}

Petroleum-derived chemicals have been essential in modern society because of their high demand in manufacturing fuels, solvents and materials among others. To cope with the grand concerns associated with global climate change and limited availability of fossil resources in the future, there has been much effort exerted to develop microbial strains capable of producing diverse chemicals and materials from renewable resources. Aromatic chemicals are important in chemical, food, polymer and pharmaceutical industries since they serve various purposes $[1,2]$. While bio-based processes for the production of a few aromatics have been commercialized [3], the majority of aromatic compounds are chemically synthesized due to the inefficiency of their biological production or even the lack of an appropriate bioproduction

\footnotetext{
${ }^{*}$ Correspondence: leesy@kaist.ac.kr

${ }^{1}$ Metabolic and Biomolecular Engineering National Research Laboratory,

Department of Chemical and Biomolecular Engineering (BK21 Plus Program) and Institute for the BioCentury, Korea Advanced Institute of Science and Technology (KAIST), Daejeon 34141, Republic of Korea Full list of author information is available at the end of the article
}

process. With the rapid advances in systems metabolic engineering tools and strategies, however, microbial production of aromatic compounds has seen a considerable progress over the past few years [4]. By this approach, chemical processes for the synthesis of various aromatics using petroleum-derived benzene, toluene and xylene (BTX) as the starting materials can be replaced by biobased sustainable and environmentally friendly processes using renewable non-food resources.

The chemicals that have been produced by microorganisms can be classified as natural (native or non-native) and non-natural ones [5, 6]. Among aromatic compounds produced in microbial systems, for example, the plantoriginated aromatics such as phenolic acids, flavonoids, stilbenoids, coumarins and their derivatives are natural chemicals but non-native to many microorganisms [7]. On the contrary, some other common aromatic chemicals including cis,cis-muconic acid and styrene are nonnatural chemicals. Since the vast majority of microbially produced aromatic chemicals are derived from shikimate (SHK) and aromatic amino acids including L-phenylalanine (L-PHE), L-tyrosine (L-TYR) and L-tryptophan 
(L-TRP), we herein categorize these aromatic compounds into (i) intermediates and derivatives of the SHK pathway, and (ii) aromatic amino acids (L-PHE, L-TYR and L-TRP) and their derivatives, on the basis of different biosynthetic pathway modules from which they are derived (Fig. 1).

In this paper, we review the current status of microbial production of various aromatic compounds focusing on metabolic engineering tools and strategies employed. In particular, those aromatic compounds that have been produced in engineered microbial cell factories from renewable feedstocks are described according to the different biosynthetic modules, with highlights on the construction of biosynthesis pathways, enzyme engineering, modulation of metabolic fluxes, omics- and modeling-based technologies. These strategies and design principles can also be implemented in the future for microbial production of new and more complex aromatic compounds.

\section{Engineering the central carbon metabolism for aromatic precursors}

Metabolic intermediates in the SHK pathway and in the aromatic amino acid biosynthesis pathway are good precursors for the production of various aromatic compounds. However, these metabolites do not typically accumulate during the metabolic reactions due to the tight regulation. To enhance metabolic fluxes towards the SHK and aromatic amino acid pathways, several welldefined conventional strategies that have been employed include: (i) replacing the native phosphotransferase system (PTS)-mediated glucose uptake system with alternatives, (ii) increasing availability of the two key SHK pathway precursors, phosphoenolpyruvate (PEP) and erythrose-4-phospahte (E4P), (iii) down-regulating carbon fluxes towards competing and catabolic pathways of precursors, and (iv) enhancing the bottleneck enzyme reactions.

Phosphoenolpyruvate is considered a rate-controlling precursor in the SHK pathway since it is also needed for the PTS system responsible for the uptake and phosphorylation of particular sugars and sugar derivatives and also for the biosynthesis of cellular materials [8]. To decrease PEP consumption by PTS during sugar uptake, alternative carbon sources such as xylose and L-arabinose that do not require PTS for their uptake have been employed. It was reported that the use of xylose as a sole carbon source resulted in production of $820.18 \mathrm{mg} / \mathrm{L}$ of 4-hydroxymandellic acid (4-HMA) in an engineered Escherichia coli, whereas the use of glucose

(See figure on next page.)

Fig. 1 De novo biosynthesis of various aromatic compounds derived from the SHK and aromatic amino acid biosynthesis pathway. Abbreviations for metabolites: 4-HPPA: 4-hydroxyphenylpyruvate; AA: anthranilic acid; ABC: ATP-binding cassette transporter; L-ARA: L-arabinose; CHA: chorismate; CLB: cellobiose; DAHP: 3-deoxy-D-arabinoheptulosonate 7-phosphate; DHAP: dihydroxyacetone phosphate; DHQ: 3-dehydroquianate; DHS: 3-dehydroshikimate; E4P: erythrose 4-phosphate; EPSP: 5-enolpyruvyl-shikimate 3-phosphate; FBP: fructose 1,6-biphosphate; G3P: glyceraldehyde 3-phosphate; G6P: glucose 6-phosphate; GLC: glucose; GLY: glycerol; PCA: protocatechuic acid; PEP: phosphoenolpyruvate; L-PHE: L-phenylalanine; PP: pentose phosphate; PPA: phenylpyruvate; PTS: phosphotransferase system; PYR: pyruvate; S3P: shikimate-3-phosphate; SHK: shikimate; SMP: proton symporter; TCA: tricarboxylic acid; L-TRP: L-tryptophan; L-TYR: L-tyrosine; X5P: xylose 5-phosphate; XYL: xylose. Abbreviations for enzymes: 4ADCL: 4-amino-4-deoxychorismate lyase; 4-CL: 4-coumarate:CoA ligase; AAAH: aromatic amino acid hydroxylase; AAS: aromatic acetaldehyde synthase; AAT: aromatic amino acid transaminase; ACAR: aromatic carboxylic acid reductase; ADCS: aminodeoxychorismate synthase; ADH: alcohol dehydrogenase; AntABC: anthranilate 1,2-dioxygenase; AROE: shikimate dehydrogenase; AS: hydroquinone glucosyl transferase; BDC: 2,3-DHBA decarboxylase; C3H: -coumarate 3-hydroxylase; $\mathrm{C4H}$ : cinnamic acid decarboxylase; CAR: carboxylic acid reductase; CCR: cinnamoyl-CoA reductase; $\mathrm{CDO}$ : catechol 1,2-dioxygenase; $\mathrm{CHI}$ : chalcone isomerase; CHS: chalcone synthase; Comt: caffeate O-methyltransferase; COMT: catechol-O-methyltransferase; CPR: cytochrome P450 reductase; CS: chorismate synthase; DAT: D-amino acid transferase; DBR: double bond reductase; DDC: L-DOPA decarboxylase; DHAPS: 3-deoxy-D-arabinoheptulosonate 7-phosphate synthase; DHQD: 3-dehydroquianate dehydratase; DHQS: 3-dehydroquianate synthase; DHS-DH: 3-dehydroshikimate dehydratase; Dmd: D-mandelate dehydrogenase; ECH: feruloyl-CoA hydratase/ lyase; EntA: 2,3-dihydro-2,3-dihydroxybenzoate dehydrogenase; EntB: isochorismatase; EntC: isochorismate synthase; EntD: phosphopantetheinyl transferase; EPSPS: 5-enolpyruvyl-shikimate 3-phosphate synthase; ER: enoate reductase; F3H: flavanone 3-hydroxylase; F3'H: flavonoid 3-hydroxylase; F3'5'H: F3'5' H-encoded 3', 5'-hydroxylase; FCS: feruloyl-CoA synthetase; FDC: ferulate decarboxylase; FLDH: D-phenyllactate dehydrogenase; FLS: flavanol synthase; FMO: flavin-containing monooxygenase; FNS: flavone synthase; GT2: gallic acid glucosyltransferase; GTF: glucosyltransferase; HA-DH: hydroxyacyl-dehydrogenase; HmaS: L-4-hydroxymandelate synthase; Hmo: L-4-hydroxymandelate oxidase; HPAH: 4-hydroxyphenylacetate 3-hydroxylase; HpgAT: L-4-(hydroxyl)-phenylglycine aminotransferase; HpgT: L-4-hydroxyphenylglycine aminotransferase; ICS: isochorismate synthase; IDH: indole acetic acid dehydrogenase; IpdC: indole-3-pyruvic acid decarboxylase; IPL: isochorismate pyruvate lyase; D-LDH: D-lactate dehydrogenase; L-LDH: L-lactate dehydrogenase; MNX1: 4-hydroxybenzoate 1-hydroxylase; PABAS: $p$-aminobenzoate synthase; Pad: phenolic acid decarboxylase; PAD: phenylacrylate decarboxylase; PAL: phenylalanine ammonia lyase; PCD: protocatechuic acid 4,5-dioxygenase; PHBD: 4-hydroxybenzoate decarboxylase; PHBH: $p$-hydroxybenzoate hydroxylase; QAD: quinate dehydrogenase; RAS: rosmarinic acid synthase; SDC: salicylate decarboxylase; SK: shikimate kinase; SMO: salicylate monooxygenase; STS: stilbene synthase; T-5H: tryptamine 5-hydroxylase; TAL: tyrosine ammonia-lyase; TDC: tryptophan decarboxylase; Tnase: tryptophanase; TPH: tryptophan 5-hydroxylase; TPL: tyrosine phenol-lyase; TrpE: anthranilate synthase; TYDC: tyrosine decarboxylase; TYO: tyramine oxidase; UbiC: chorismate lyase; VioA: L-tryptophan oxidase; VioB: iminophenyl-pyruvate dimer synthase; VioC: violacein synthase; VioD: protoviolaceinate synthase; VioE: violacein biosynthesis protein. Continuous arrows show single enzymatic reactions, and dashed arrows show multiple enzymatic reactions 


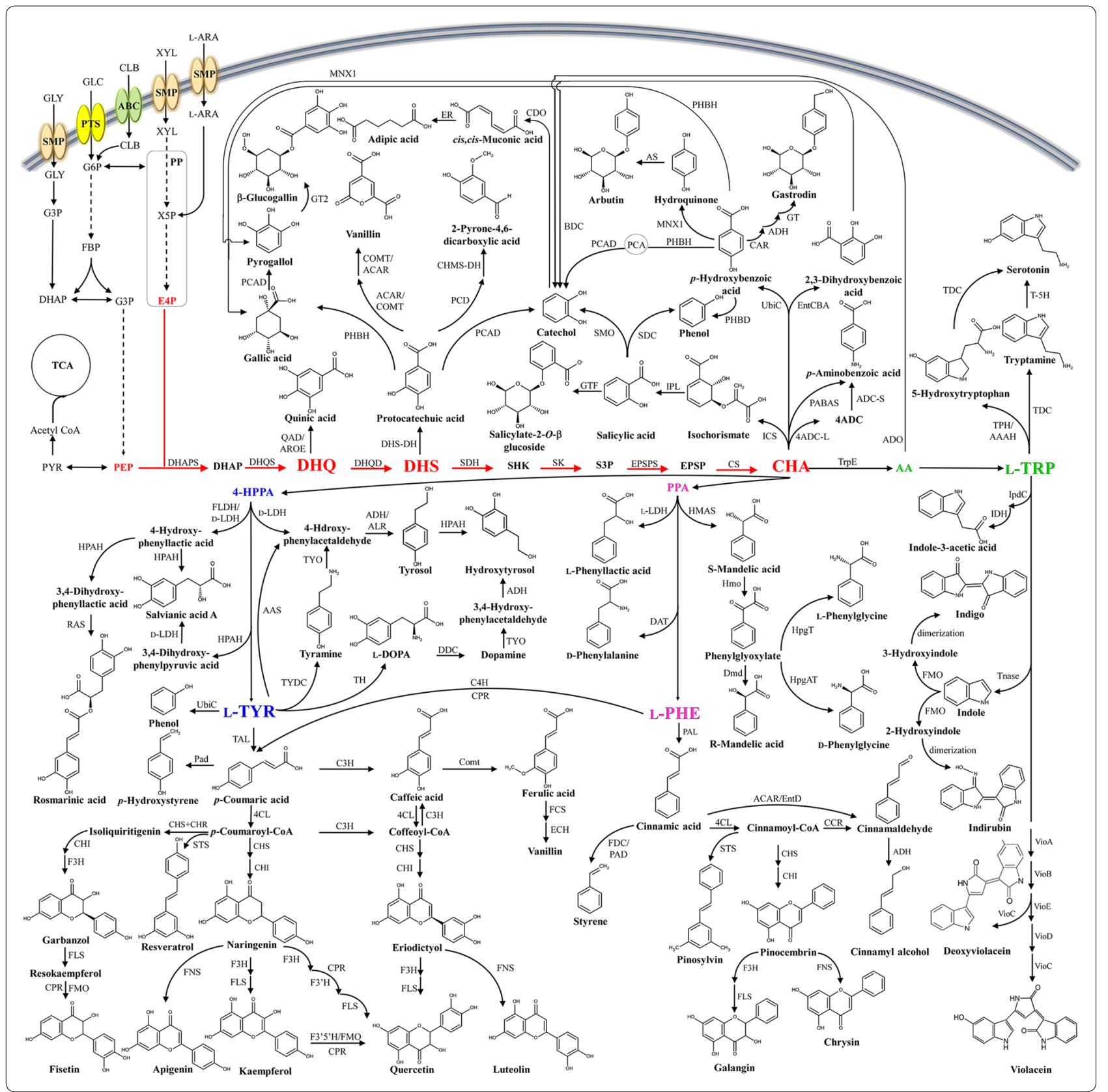

yielded $747.13 \mathrm{mg} / \mathrm{L}$ 4-HMA [9]. Also, the use of a nonPTS system which does not consume PEP is an alternative way for sugar transport. For example, through the facilitated diffusion with ATP-based phosphorylation, glucose can be first transported by galactose permease (GalP), and then phosphorylated by glucokinase in $E$. coli [10]. In a mutant $E$. coli strain lacking PTS, the carbon flux was successfully increased towards aromatic amino acid pathway due to the elevated glucokinase activity leading to rapid glucose consumption, although the specific cell growth rate was lower than that of the wild-type strain [11]. To investigate the effect of nonPTS system on production of 3-deoxy-D-arabinoheptulosonate 7-phosphate (DAHP) as a starting precursor in the SHK pathway, the performance of 3-dehydroquinate (DHQ) synthase-deficient $E$. coli strains having PTS and non-PTS were compared. In the E. coli strain having the non-PTS system, the yield of DAHP on glucose was 1.65-fold higher than that obtained with the E. coli strain having the PTS system [12]. Moreover, the use of 
a non-PTS system together with overexpression of several key genes that encode DAHP synthase, transketolase and chorismate (CHA) mutase-prephenate dehydratase, resulted in a L-PHE overproducer strain of $E$. coli with the yield of $0.33 \mathrm{~g} / \mathrm{g}$ glucose [13]. The non-PTS system of Zymomonas mobilis was also used to improve the cellular PEP availability in combination with the native nonPTS system in E. coli. In particular, the glucose facilitator encoded by $g l f$ in $Z$. mobilis transports glucose by facilitated diffusion, whereas that encoded by galP in E. coli transports glucose by proton symport system. Glucose transport by both systems is followed by the glucokinases using ATP-based phosphorylation. When compared to the native non-PTS in E. coli, the heterologous Z. mobilis non-PTS system was more efficient since it enabled production of $60 \mathrm{~g} / \mathrm{L}$ DHS with higher productivity [14]. Besides the well-known non-PTS systems, a new nonPTS system was recently reported as myo-inositol transporter (encoded by iolT1) in Corynebacterim glutamicum [15]. Through deletion of the transcriptional regulator protein encoded by iolR that inhibits the expression of iol gene cluster, the non-PTS system of C. glutamicum was activated, leading to production of $4.01 \mathrm{~g} / \mathrm{L}$ of cis,cismuconic acid with the highest yield of $22 \% \mathrm{~mol} / \mathrm{mol}$ glucose in E. coli [16].

To increase the PEP pool, several other useful strategies employed include: overexpression of PEP-forming enzymes (i.e., PEP synthase and PEP carboxykinase) or inactivation of PEP-degrading enzymes (i.e., pyruvate kinases and PEP carboxylase) [3]. Although the inactivation of PEP-degrading enzymes modulated carbon flux towards the SHK pathway, cell growth decreased by almost a half probably due to the accumulation of byproducts such as acetate and pyruvate and decrease of TCA intermediates. On the other hand, the increase in the PEP synthase activity positively affected aromatic compound production, especially when transketolase was also overexpressed [17].

Increase of the E4P pool also improves formation of DAHP. Since the first studies that showed the contribution of E4P to the synthesis of DAHP [18] and aromatic compounds [19], its overexpression has been one of the strategies popularly used in aromatic compounds production. As an intermediate of the pentose phosphate (PP) pathway, E4P is synthesized from sedo-heptulose1,7-biphosphate [20].

\section{Engineering the SHK pathway and its derivatives}

The SHK pathway links the central carbon metabolism to the biosynthesis of aromatic amino acids including L-TRP, L-TYR and L-PHE [21]. This pathway comprising seven successive enzymatic reactions leads to the biosynthesis of CHA, a key aromatic precursor, which is also a branch point for the biosynthesis of three aromatic amino acids as well as diverse aromatic compounds [22, 23]. Intermediates in the SHK pathway are also precursors for the biosynthesis of diverse secondary metabolites especially in plants [24]. As these intermediates are important for the biosynthesis of various derivative aromatic compounds, development of strategies for their efficient production is a key for the de novo synthesis of numerous aromatic compounds.

Since the first chemical synthesis of $p$-hydroxybenzoic acid (PHBA) from benzene on industrial scale in 1980's, PHBA and its derivatives have been extensively used for the synthesis of liquid crystal, paraben (a preservative used in cosmetic and pharmaceutical products), phenol and others $[25,26]$. Alternatively, PHBA is naturally produced through one-step conversion from $\mathrm{CHA}$ by the ubiC-encoded $\mathrm{CHA}$ pyruvate-lyase during the synthesis of ubiquinone in $E$. coli, or through cascade reactions from CHA through phenylpropanoids, i.e., $p$-coumaric acid, in the plant secondary metabolism [25, 27]. By mimicking this natural pathway of $E$. coli, metabolically engineered $C$. glutamicum was able to produce PHBA to the highest titer and yield of $36.6 \mathrm{~g} / \mathrm{L}$ and $41 \%$ (mol/ mol), respectively [26], which were higher than those achieved with engineered E. coli [25]. Apart from the conventional metabolic engineering strategies such as the elimination of competing pathways through inactivation of enzymes involved in the central carbon metabolism (i.e., ldh $A$-encoded lactate dehydrogenase, $p y k$-encoded pyruvate kinase, $h d p A$-encoded haloacid dehalogenase) and in SHK pathway ( $q s u B$-encoded dehydroshikimate dehydratase, qsuD-encoded shikimate dehydrogenase, pobA-encoded 4-hydroxybenzoate hydroxylase), the use of PHBA-resistant UbiC from Providencia rustigianii and growth-arrested bioprocess proved to be effective for increasing PHBA production in the engineered C. glutamicum. Additionally, a mutated aroG gene from $E$. coli and the native aroCKB that encodes CHA synthase, SHK kinase and DHQ synthase, respectively, were overexpressed via chromosomal integration [26]. In $E$. coli, the enhanced central carbon flux towards CHA was accomplished by overexpressing transketolase in PP pathway and feedback insensitive DAHP synthase $\left(\right.$ AroF $\left.^{\mathrm{fbr}}\right)$, DHQ synthase, SHK kinase, 5-enolpyruvylshikimate 3-phosphate (EPSP) synthase and CHA synthase in SHK pathway [25]. Although Saccharomyces cerevisiae can tolerate high concentration of PHBA up to $38.3 \mathrm{~g} / \mathrm{L}$ [28], the engineered yeast produced PHBA only to milligram levels under either batch operation in shake-flask or pulsed-feeding in fermenter mainly due to excessive byproduct formation $[28,29]$. An E. coli-E. coli co-culture system has also been developed for PHBA production. The E. coli strain harboring the upstream PHBA pathway 
was engineered to produce and secrete DHS, which was assimilated by the engineered $E$. coli strain harboring the downstream pathway through the DHS importer ShiA to produce PHBA. Also, the sugar utilization pathways in both strains were manipulated for independent carbon source utilization. When these two E. coli strains were co-cultured, $2.3 \mathrm{~g} / \mathrm{L}$ of PHBA was produced from a mixture of glucose and xylose [30].

cis,cis-Muconic acid is a dicarboxylic acid used as a precursor in the chemical synthesis of adipic acid, a major building block for the synthesis of nylon. Currently, cis,cis-muconic acid is produced by petrochemical processes. Biological production of cis,cis-muconic acid has also been developed, either through biotransformation of aromatic substrates (e.g., benzoate) or via de novo biosynthesis from renewable sources (e.g., lignin and glucose) [31]. On the basis of its presence as an intermediate metabolite both in the $\beta$-ketoadipate pathway during aromatic hydrocarbon degradation in bacteria [32-34] and the ubiquinone biosynthesis pathway [35], cis,cismuconic acid has been biosynthesized through two different pathways using DHS or CHA as a SHK pathway precursor [36]. After the first report on microbial production of cis,cis-muconic acid employing DHS as precursor [37], the strategies have been mainly focused on optimizing the heterologous pathway comprising DHS dehydratase, PCA decarboxylase and catechol 1,2-dioxygenase. The highest titer of cis,cis-muconic acid reported is $36.8 \mathrm{~g} / \mathrm{L}$ using an engineered $E$. coli harboring the aroZ-encoded DHS dehydratase and aroY-encoded PCA decarboxylase from Klebsiella pneumonia, and cat $A$-encoded catechol 1,2-dioxygenase from Acinetobacter calcoacetius [38]. By using the same metabolic engineering strategy with DHS as precursor, 2-pyrone4,6-dicarboxylic acid has also been produced. The highest 2-pyrone-4,6-dicarboxylic acid titer of $16.7 \mathrm{~g} / \mathrm{L}$ was achieved by combining the downstream heterologous bioconversion steps catalyzed by asbF-encoded DHS dehydratase from Bacillus thuringiensis, pmdABencoded PCA 4,5-dioxygenase and pmdC-encoded 4-carboxy-2-hydroxymuconate-6-semialdehyde dehydrogenase from Comamonas testosteroni [39]. Using an $E$. coli-E. coli co-culture system, $4.7 \mathrm{~g} / \mathrm{L}$ of cis,cis-muconic acid was produced from a glucose/xylose mixture [30].

While earlier studies mainly focused on producing cis,cis-muconic acid from DHS, the recent studies employed CHA derivatives as precursors, i.e., anthranilic acid, 2,3-dihydroxybenzoic acid (2,3-DHBA), PHBA, salicylic acid [40-43]. Among them, the pathway derived from salicylic acid yielded gram-level of cis,cis-muconic acid in shake-flask experiments [43], whereas the production levels by using the other precursors remained quite low. For instance, the biosynthetic pathway of cis,cis-muconic acid derived from anthranilic acid was inspired by the anthranilate degradation pathway followed by subsequent formation of catechol and cis,cismuconic acid in Actinobacter and Pseudomonas [40]. The Rhizobium radiobacter 2,3-DHBA decarboxylase (BDC) was also reported to convert 2,3-DHBA to catechol through its promiscuous activity [41]. Additionally, S. cerevisiae has also been engineered for biosynthesis of cis,cis-muconic acid from DHS by introducing candidate genes from different organisms, but cis,cis-muconic acid production was rather inefficient (Table 1) $[28,44$, 45]. When comparing the recent microbial platforms for cis,cis-muconic acid production, one remarkable example is the engineered Pseudomonas putida strain that showed enhanced activity of aroY-encoded PCA decarboxylase from Enterobacter cloacae, resulting from co-expression with other small proteins called EcdB and EcdD. These proteins led to the conversion of PCA to catechol rather than the intermediate in $\beta$-ketoadipate pathway [46]. By combining synthetic cis,cis-muconic acid pathway with further bioconversion reaction catalyzed by enoate reductase (ER) from Bacillus coagulans, microbial production of adipic acid was achieved in engineered S. cerevisiae, although the final titer of adipic acid was low [47].

Vanillin (4-hydroxy-3-methoxybenzaldehyde), a widely used fragrance and aroma agent in various industries, is one of the first aromatic compounds produced by engineered microbial systems. Its microbial production is considered as an eco-friendly alternative to the traditional methods (i.e., extraction from seedpods of orchids and lignin/hydrocarbon (guaiacol)-based synthesis) as reviewed elsewhere [48]. While vanillin is intrinsically synthesized as a secondary metabolite in Vanilla planifolia through pathways not fully understood [49], a few actinomycetes, such as Streptomyces setonii 75vi2 (recently known as Amycolatopsis sp. strain ATCC 39116) and Amycolatopsis sp. HR167, are also capable of producing vanillin as an intermediate in their ferulic acid degradation pathway [50, 51]. This degradation pathway can vary with initial reactions in different microorganisms, which has been previously reviewed in detail [49]. In addition, Pseudomonas sp. strain HR199 was also reported to synthesize vanillin from ferulic acid and eugenol by the contribution of native enzymes such as $f c s$-encoded feruloyl-CoA synthetase, ech-encoded enoyl-CoA hydratase/aldolase and aat-encoded B-ketothiolase [52]. This pathway is based on the so-called CoA-dependent retro-aldol mechanism, and it is inherently present in a number of microorganisms, i.e., Delftia acidovorans [53], Pseudomonas fluorescens AN103, Amycolaptosis sp. strain HR167 [49] and P. putida [54]. Metabolic engineering of a native vanillin producer $P$. $f l u$ orescens led to relatively high-level production of vanillin 


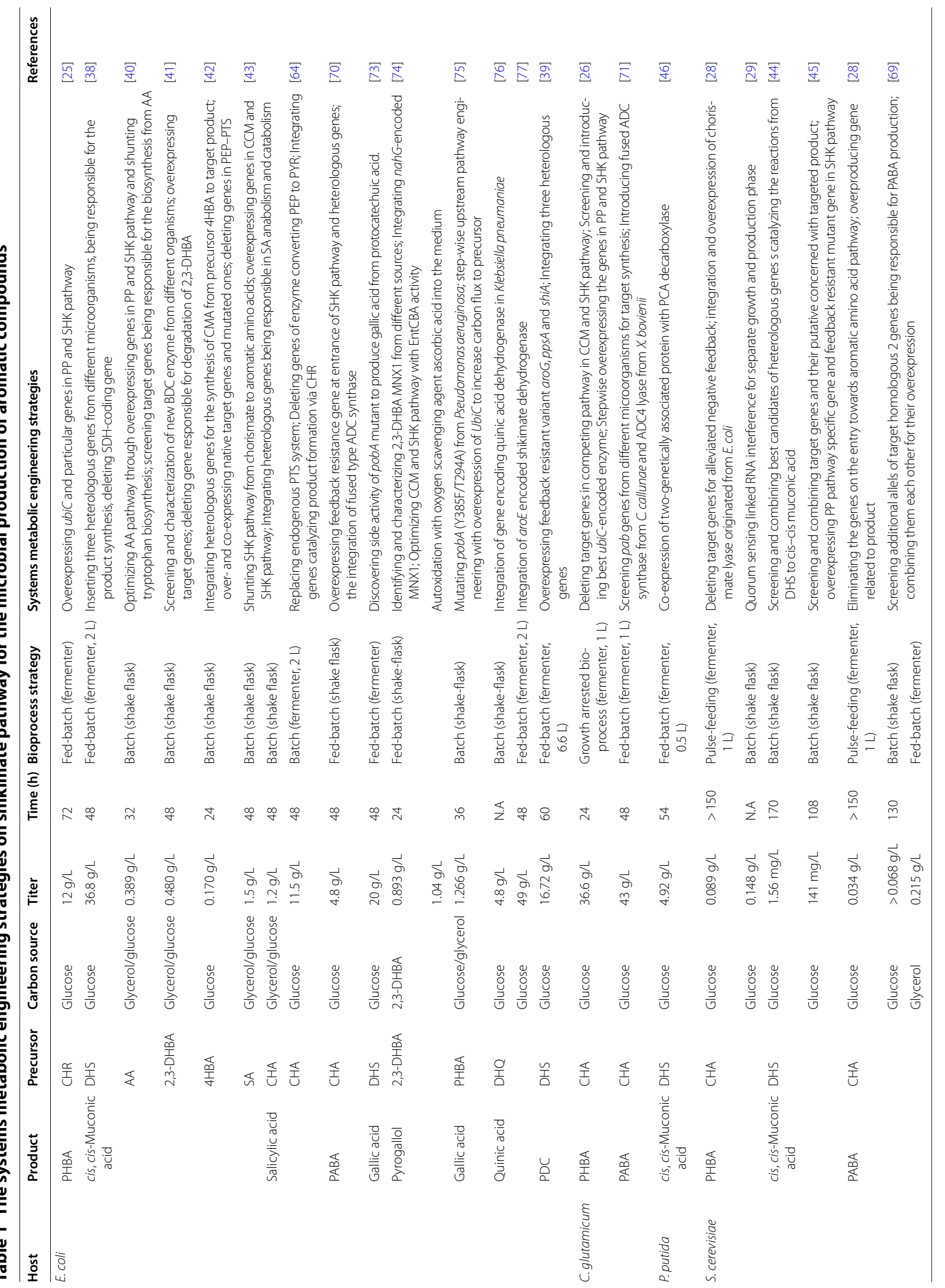


from ferulic acid [55]. On the other hand, a recombinant strain of E. coli XL1-Blue harboring the corresponding heterologous genes $f c s$ and ech was also developed for vanillin production from ferulic acid. The vanillin titer was further improved to $5.14 \mathrm{~g} / \mathrm{L}$ in shake-flask experiments by reducing the acetyl-CoA consumption through overexpression of gltA (encoding citrate synthase) and deletion of $i c d A$ (encoding isocitrate dehydrogenase) [56]. In another report, $7.8 \mathrm{~g} / \mathrm{L}$ of vanillin was produced from ferulic acid by a two-stage process using $E$. coli whole-cell biocatalysts harboring coenzyme-independent decarboxylase/oxygenase [57]. Despite high titers of vanillin, the requirement of the expensive substrate ferulic acid is a drawback associated with the aforementioned biotransformation processes. To replace ferulic acid with a relatively inexpensive substrate, such as eugenol, an engineered $E$. coli was developed by introducing vaoAencoded vanilly alcohol oxidase from Penicillium simplicissimum. This strain was able to produce $0.3 \mathrm{~g} / \mathrm{L}$ of vanillin through two-step biotransformation process [58]. In addition to biotransforming ferulic acid, eugenol and isoeugenol for vanillin production, a number of studies have shown de novo vanillin production from renewable and even cheaper substrates such as glucose. In an initial study, an engineered E. coli strain expressing the aroZencoded DHS dehydratase from the mold fungus Podospora anserine and human catechol-O-methyltransferase (COMT) was used to first produce vanillic acid from glucose. This was followed by further reduction of vanillic acid to vanillin by whole-cell catalysis of Neurospora crassa having the required activity of aromatic carboxylic acid reductase (ACAR) [59]. Thereafter, the whole synthetic vanillin pathway was successfully reconstructed in both Schizosaccharomyces pombe and S. cerevisiae strains using the Nocardia iowensis ACAR, which eliminated the need for a separate whole-cell catalysis step. Moreover, the C. glutamicum phosphopantetheinyl transferase was co-expressed in the engineered S. cerevisiae strain for activation of ACAR, and endogenous alcohol dehydrogenases in the S. cerevisiae host were deleted to prevent reduction of vanillin to vanilly alcohol. In the engineered $S$. pombe, vanillin production was enhanced by introducing the Arabidopsis thaliana UDP glycosyltransferase that converts vanillin to $\beta$-D-glucoside. The use of $\beta$-Dglucoside could reduce the toxicity caused by vanillin at high concentrations, and the microbially produced $\beta$-Dglucoside can be subsequently converted back to vanillin through enzymatic conversion [60]. Through deletion of the pyruvate decarboxylase-encoding gene that was suggested by in silico genome-scale metabolic simulation, the vanillin $\beta$-D-glucoside production could be improved to $500 \mathrm{mg} / \mathrm{L}$ in engineered $S$. cerevisiae, which was fivefold higher than that obtained with engineered $S$. pombe [61].

Salicylic acid (2-hydroxybenzoic acid) is widely used in the pharmaceutical industry owing to its analgesic, antiinflammatory and antipyretic activities [62]. In nature, it is synthesized in plants and bacteria to serve as a signal molecule and a precursor in the production of ironchelating siderophore, respectively [63]. Even though the salicylic acid biosynthetic pathway has been well studied previously, only recently the highest salicylic acid titer of $11.5 \mathrm{~g} / \mathrm{L}$ was achieved via CHA in engineered E. coli, in which the native PTS was replaced by GalP/Glk system and the downstream glycolytic pathway was blocked to enhance the precursors towards SHK pathway [64]. Apart from E. coli, C. glutamicum was also engineered to produce salicylic acid to a concentration of $100 \mathrm{mg} / \mathrm{L}$ in shake-flask experiments by introducing the irp9-encoded bifunctional isochorismate synthase/isochorismate pyruvate lyase from Yersinia enterocolitica (Table 1) [65]. The glycosylated form of salicylic acid, salicylate 2-O- $\beta$ D-glucoside (SAG), was also successfully produced in engineered $E$. coli by the addition of the $A$. thaliana glucosyltransferase to the existing salicylic acid biosynthetic pathway [66]. The SAG titer of $2.5 \mathrm{~g} / \mathrm{L}$ was achieved by using a co-culture system. In this system, one recombinant $E$. coli strain harboring the upstream salicylic acid pathway was used to produce salicylic acid, which was converted to SAG by the second E. coli strain expressing a codon-optimized $A$. thaliana glucosyltransferase gene [67].

$p$-Aminobenzoic acid (PABA) is a widely used compound in pharmaceutical, resin and dye industries, and is currently produced from petroleum-derived toluene. PABA is also a natural metabolite in the folate biosynthesis in plants and bacteria synthesized through a two-step conversion of CHA catalyzed by aminodeoxychorismate (ADC) synthase and 4-amino-4-deoxychorismate (4ADC) lyase [68]. The first microbial production of PABA was achieved in $S$. cerevisiae by overexpressing $A B Z 1$-encoded PABA synthase from another S. cerevisiae species and also by deleting $A R O 7$ (encoding $\mathrm{CHA}$ mutase) and TRP3 (encoding indole-3-glycerolphosphate synthase/anthranilate synthase complex) in the aromatic amino acid pathway [28]. Using this engineered $S$. cerevisiae strain, the effects of different carbon sources (glucose, glycerol and glycerol/ethanol mixture) on PABA production were also investigated based on the results obtained from in silico analysis. Among the carbon sources examined, the highest PABA titer and yield reached $215 \mathrm{mg} / \mathrm{L}$ and $2.64 \% \mathrm{~mol} / \mathrm{mol}$ carbon, respectively, using glycerol/ethanol mixture [69]. Compared to the engineered yeast, engineered $E$. coli harboring $p a b A$ - and $p a b B$ (encoding heterodimer complex ADC 
synthase), pabC (encoding 4ADC lyase), and E. coli $a r o F^{f b r}$ (encoding feedback resistant AroF) produced $4.8 \mathrm{~g} / \mathrm{L}$ of PABA by fed-batch fermentation [70]. The highest PABA titer of $43 \mathrm{~g} / \mathrm{L}$ was achieved by an engineered $C$. glutamicum harboring the best combination of Corynebacterium callunae pabAB and Xenorhabdus bovienii pabC in fed-batch culture [71].

Pyrogallol, also known as 1,2,3-trihydroxybenzene, is an important platform chemical used in food, pharmaceutical and polymer industries. Currently it is chemically synthesized from gallic acid in the presence of $\mathrm{HCl}$, where gallic acid is obtained by enzymatic conversion of gallotannin (tannic acid) using tannese [72]. However, this process for commercial production of pyrogallol is limited due to the low yield of gallotannin obtained by plant extraction. The characterization of genes that encode tannese and gallic acid decarboxylase in a number of microorganisms, such as Streptococcus gallolyticus, Lactobacillus plantarum and Pantoea agglomerans, pioneered the research on microbial production of pyrogallol. Starting with DHS in SHK pathway, PCA and gallic acid were sequentially synthesized in engineered $E$. coli by aroZ-encoded DHS dehydratase from $K$. pneumoniae and mutated pobA-encoded PHBA hydroxylase from Pseudomonas aeruginosa, respectively. Using this engineered $E$. coli, the highest reported gallic acid titer of $20 \mathrm{~g} / \mathrm{L}$ was achieved from glucose in fed-batch fermentation. To produce pyrogallol from gallic acid, this $E$. coli strain was further engineered by introducing aroY (encoding PCA decarboxylase) from $K$. pneumonia since the desired enzymatic activity for this conversion was shown by non-oxidative decarboxylase isolated from Pantoea agglomerans. However, the pyrogallol production was not detected [73]. In recent study, pyrogallol has been synthesized from CHA via 2,3-DHBA catalyzed by 2,3-DHBA synthase and 2,3-DHBA 1-monoxygenase. E. coli overexpressing the native 2,3-DHBA synthase and an efficient 2,3-DHBA 1-monoxygenase (encoded by nahG) from P. putida produced $201.5 \mathrm{mg} / \mathrm{L}$ of pyrogallol from glucose. Pyrogallol production by this recombinant strain was further improved to $1035.8 \mathrm{mg} / \mathrm{L}$ by increase of flux to SHK pathway, modular pathway optimization and reduction in pyrogallol autoxidation [75]. Moreover, $1266.4 \mathrm{mg} / \mathrm{L}$ of gallic acid was also produced via CHA by engineered $E$. coli harboring a highly efficient mutated PobA from Pseudomonas aeruginosa in shake flasks [76].

Quinic acid (QA), commonly used as synthons in pharmaceutical industry, is currently produced from phenol or benzene by petrochemical processes. QA is among the first produced cyclohexane carboxylic acids by engineered microorganisms with the discovery of its catabolism in K. pneumoniae. QA production from glucose was first achieved in an engineered $E$. coli expressing the
K. pneumoniae qad gene encoding QA dehydrogenase [76]. In another study, overexpression of the E. coli aroE encoding the native SHK dehydrogenase and knocking out aro $D$ encoding DHQ dehydratase resulted in production of $49 \mathrm{~g} / \mathrm{L}$ QA from glucose by fed-batch culture [77]. Based on the biosynthesized QA in these studies, downstream chemical processes were also developed to produce other important industrial quinone derivatives such as benzoquinone and hydroquinone [77, 78].

Arbutin, a glycosylated hydroquinone in plant metabolism, finds applications in cosmetic industry due to its whitening activity that inhibits tyrosinase-mediated melanogenesis. It also serves as an anti-microbial, antiinflammatory and anti-oxidant agent. Compared to chemical processes, enzymatic methods for arbutin production is more favorable due to the regioselectivity, mild operation conditions and use of fewer toxic chemicals. In this regard, various plant- and microbial-based approaches have been investigated for enzymatic production of arbutin from hydroquinone using a series of glycosylation enzymes originated from different organisms [78]. De novo biosynthesis of arbutin from glucose was achieved for the first time in an engineered $E$. coli strain expressing the genes encoding the flavin adenine dinucleotide-dependent 4-hydroxybenzoate 1-hydroxylase (MNX1) from Candida parapsilosis and uridine phosphate glucose (UPDG)-based hydroquinone glucosyl transferase (AS) from Rauvolfia serpentine. Further enhancement of the SHK pathway flux and optimization of initial glucose concentrations improved arbutin production up to $4.2 \mathrm{~g} / \mathrm{L}$ in shake flasks [79]. In a recent study, the highest reported arbutin titer of $6.79 \mathrm{~g} / \mathrm{L}$ was achieved by engineered Pseudomonas chlororaphis expressing the aforementioned MNX1 and AS genes from the genome [80].

Gastrodin, a phenolic glycoside found in plants, serves as a drug substance based on its sedative, hypnotic, anticonvulsive and neuroprotective activities. It is currently produced by either chemical synthesis or extraction from plants. In nature, some organisms (e.g., Rhizopus chinensis) have the ability to transform $p$-hydroxybenzaldehyde to gastrodin [81]. Although the natural gastrodin biosynthesis pathway in plants has not been fully identified, de novo production of gastrodin from glucose has been reported in engineered E. coli harboring an artificial metabolic pathway. This synthetic pathway was derived from PHBA, which was further converted by the Nocardia carboxylic acid reductase, endogenous alcohol dehydrogenases and an engineered Rhodiola glycosyltransferase. The engineered $E$. coli overexpressing the pathway genes produced $545 \mathrm{mg} / \mathrm{L}$ of gastrodin [82]. 
A number of aromatic amines have also been produced from glucose by expanding the SHK pathway via CHA in E. coli host (Fig. 2). The key step of the artificial biosynthesis of these aromatic amines was the formation of 4-aminophenylpyruvate from CHA by the sequential activities of 4-amino-4-deoxychorismate synthase (encoded by papA), 4-amino4-deoxychorismate mutase (encoded by papB) and 4-amino-4-deoxyprephenate dehydrogenase (encoded by $p a p C$ ) from Streptomyces venezuelae and Streptomyces pristinaespiralis. Subsequently, this biosynthetic pathway was branched into 4-aminophenylacetaldehyde and 4-aminophenylalanine by the reactions catalyzed by phenylpyruvate decarboxylase and aminotransferase, respectively. In the last step, 4-aminophenylacetic acid and 4-aminophenylethanol were produced from 4-aminophenylacetaldehyde, while 4-aminocinnamic acid and 4-aminophenylethylamine were produced from 4-aminophenylalanine [83].

\section{Engineering the biosynthesis pathway of aromatic amino acids and their derivatives}

Three aromatic amino acids, L-PHE, L-TYR and L-TRP, are derivatives of $\mathrm{CHA}$ and are synthesized through the formation of specific intermediates such as phenylpyruvate, 4-hydroxyphenylpyruvate and anthranilic acid, respectively. Hence, not only aromatic amino acids but also these intermediates play important roles as useful precursors for the production of diverse aromatic acids, alcohols, hydrocarbons, ketones, and aromatic natural compounds (mostly involved in phenylpropanoid pathway). Metabolic engineering for the production of these derivative aromatic products will be described separately according to each of the three aromatic amino acid pathways they are derived from.

\section{L-PHE pathway derivatives}

From L-PHE biosynthetic pathway, the majority of the microbially produced aromatic compounds are derived from two major precursors, phenylpyruvate and L-PHE, which are summarized in Table 2.

D-Phenylglycine (D-PHG) serves as a precursor in the production of semi-synthetic antibiotics including cephalosporin and penicillin [84], and it is either chemically produced from phenol or enzymatically synthesized by resolution of racemic mixtures originating from fine chemicals [85]. Microbial biosynthesis of D-PHG from glucose has been achieved through a three-step artificial pathway after phenylpyruvate, which consisted of the successive formation of $S$-mandelate, phenylglyoxylate and D-PHG catalyzed by hmaS-encoded 4-hydroxymandelate synthase of Amycolatopsis orientalis, hmo-encoded 4-hydroxymandelate oxidase of Streptomyces coelicolor and $h p g A T$-encoded stereoinverting hydroxyphenylglycine aminotransferase of $P$. putida, respectively. The engineered $E$. coli expressing these enzymes and with deletions of the aspartate aminotransferase-encoding asp $C$ and aromatic amino acid aminotransferase-encoding ${ }^{t y r} B$ genes produced $102 \mathrm{mg}$ D-PHG per gram dry cell weight $(\mathrm{DCW})$ from glucose [84]. Moreover, L-phenylglycine (L-PHG), a precursor in the production of several antibiotics and taxol, was also produced by replacing the HpgAT in the D-PHG pathway with L-4-hdroxyphenylglycine aminotransferase (encoded by $h p g T$ ) from $A$. orientalis and S. coelicolor. The engineered E. coli harboring these heterologous enzymes produced $51.6 \mathrm{mg} / \mathrm{g}$ DCW of L-PHG from glucose [86].

Mandelic acid is one of the aromatic fine chemicals primarily used in pharmaceutical industry and for resolution of racemic alcohols and resins. Compared to chemical methods, enzymatic synthesis of mandelic acid is more attractive as it can afford stereoisomers of mandelic acid. An engineered $E$. coli strain expressing the $A$. orientalis

(See figure on next page.)

Fig. 2 The metabolic engineering approaches to microbial production of SHK pathway derivatives. A microbial co-culture system developed for the biosynthesis of cis,cis-muconic acid from a mixture of glucose and xylose (shown in the upper panel). Metabolic design for the production of a series of chorismate-derived aromatic amines (shown in the bottom panel). Abbreviations for metabolites: 4-ACA: 4-aminocinnamic acid; 4-APAA: 4-aminophenyl acetic acid; 4-APE: 4-aminophenylethanol; 4-APEA: 4-aminophenylamine; 4-APhe: 4-aminophenylalanine; 4-APheAL: 4-aminophenyl acetaldehyde; 4-APheP: 4-aminophenyl pyruvate; AcCoA: acetyl-CoA; CA: catechol; CHA: chorismate; CMA: cis,cis-muconic acid; DAHP: 3-deoxy-D -arabino-heptulosonate 7-phosphate; DHQ: 3-dehydroquinate; DHS: 3-dehydroshikimate; E4P: erythrose 4-phosphate; F6P: fructose 6-phosphate; G6P: glucose 6-phosphate; GLC: glucose; GLY: glycerol; PCA: protocatechuic acid; PEP: phosphoenolpyruvate; L-PHE: L-phenylalanine; PYR: pyruvate; SHK: shikimate; TCA: tricarboxylic acid; L-TRP: L-tryptophan: L-TYR: L-tyrosine; X5P: xylose 5-phosphate; XYL: xylose. Abbreviations for enzymes: AAAD: aromatic amino acid decarboxylase; ADH: aldehyde dehydrogenase; ALDH: alcohol dehydrogenase; AroE: shikimate dehydrogenase; AroF: feedback sensitive DHAP synthase; AroG Gbr: feedback resistance isozyme of DHAP synthase; AroH: chorismate mutase; AT: aminotransferase; AroY: protocatechuate decarboxylase; Aroz: 3-dehydroshikimate dehydratase; CatA: catechol 1,2-dioxygenase; PapA: 4-amino-4-deoxychorismate synthase; PapB: 4-amino-4-deoxychorismate mutase; PapC: 4-amino-4-deoxyprephenate dehydrogenase; PDC: phenylpyruvate decarboxylase; PpsA: phosphoenolpyruvate synthase; ShiA: shikimate transporter, PAL: phenylalanine ammonia lyase; TktA: transketolase; YdiB: Quinate/shikimate dehydrogenase. The inactivated metabolic pathways are indicated by "X". Dotted lines indicate feedback inhibition. Native metabolic pathways are indicated by black arrows, and non-native pathways are indicated by blue arrows. Multiple metabolic reactions are indicated by sequential arrows. Blue boxes represent feedback inhibition resistant mutants of endogenous enzymes. Pink line or pink boxes represent overexpressed enzymes 


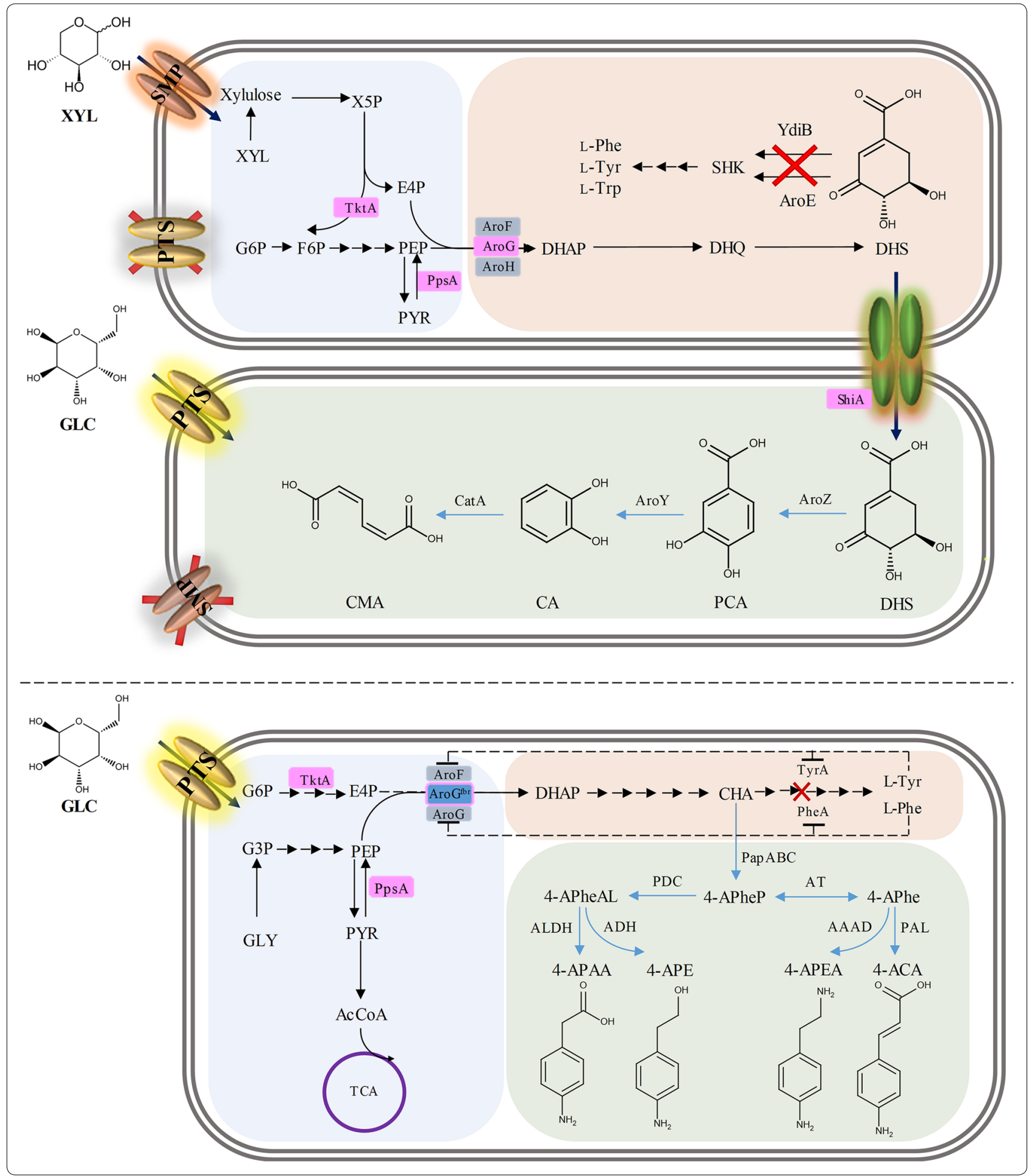

$\mathrm{HmaS}$ and with deleted competing pathways produced $0.74 \mathrm{~g} / \mathrm{L}$ of $S$-mandelate from glucose. By further expressing the S. coelicolor Hmo and D-mandelate dehydrogenase (encoded by $d m d$ ) from Rhodotorula graminis in the $S$-mandelate-producing $E$. coli, $0.68 \mathrm{~g} / \mathrm{L}$ of $R$-mandelate was produced from glucose [87]. By replacing the A. orientalis $\mathrm{HmaS}$ with a more efficient isozyme from Nocardia uniformis in an engineered S. cerevisiae, $236 \mathrm{mg} / \mathrm{L}$ of $S$-mandelate was produced from glucose [88]. 
Similar to D-PHG, D-phenylalanine (D-PHE) is also a building block in the production of semi-synthetic antibiotics such as penicillin and cephalosporin. As a L-stereoisomer, L-PHE is synthesized from phenylpyruvate by L-PHE aminotransferase, however, such an aminotransferase with D-stereoisomer activity (i.e., D-PHE aminotransferase) for producing D-PHE is still lacking. Therefore, microbial biosynthesis of D-PHE has been achieved by using the D-amino acid aminotransferase (Dat) in Bacillus species instead. Three Dats from Bacillus subtilis, Bacillus licheniformis and Bacillus amyloliquefaciens were compared through 3D structural modeling and experimental screening. When the best-performing Dat from $B$. subtilis was expressed in an L-PHE-producing E. coli, $1.72 \mathrm{~g} / \mathrm{L}$ of D-PHE was produced from glucose in bioreactor fermentation [89].

Phenyllactic acid (PhLA) is a potential monomer that can be used in the production of sustainable polyesters by chemical polymerization with other bio-based monomers [90]. Both D-PhLA and L-PhLA have been biosynthesized through one-step reduction of phenylpyruvate. An L-PHE-overproducing E. coli expressing the pprAencoded phenylpyruvate reductase from Wickerhamia fluorescens produced $29 \mathrm{~g} / \mathrm{L}$ of D-PhLA from glucose in bioreactor fermentation with optimized conditions [91]. In the same $E$. coli strain, L-PhLA was also produced from glucose by replacing PprA with the $l d h A$-encoded lactate dehydrogenase from Pediococcus acidilactici [91]. For advanced applications, D-PhLA was co-polymerized with 3-hydroxybutyrate to make novel aromatic polyesters by engineered $E$. coli through one-step fermentation. In achieving this, the pivotal steps were the choices of a suitable CoA-transferase and an engineered polyhydroxyalkonate synthase (Fig. 3) [92].

Phenylpropanoids constitute a response system in plant metabolism to protect plants against various changes in their environment. Also, they are important precursors in the production of various chemicals used in food, pharmaceutical and cosmetic industries. Biosynthesis of phenylpropanoids starts with the formation of cinnamic acid, which is a key branch point leading to the biosynthesis of various other phenylpropanoids [93]. Metabolic engineering of phenylpropanoids has been focused on cinnamic acid and its derivatives including several secondary metabolites such as flavonoids, stilbenoids, coumarins, among other phenylpropanoids [7].

Cinnamic acid (CA) is chemically synthesized either through the Perkin reaction by heating benzaldehyde and acetic anhydride with sodium acetate [94] or through the Knoevenagel condensation of malonic acid and aromatic aldehydes [95]. In plants, L-PHE is catalyzed by phenylalanine ammonia-lyase (PAL) to yield trans-CA [96]. While some PALs such as the yeast Rhodosporidium toruloides PAL possess the alternative capability to convert $\mathrm{L}$-TYR to $p$-hydroxycinnamic acid in addition to converting L-PHE to CA [97], the Streptomyces maritimus PAL shows a high substrate selectivity towards L-PHE, and it has been used for the biosynthesis of CA in engineered $E$. coli [98]. Expression of the R. toruloides PAL in a solvent-tolerant and mutated $P$. putida strain with enhanced supply of the precursor L-PHE led to production of $0.741 \mathrm{~g} / \mathrm{L}$ CA [99]. Moreover, Streptomyces lividans, well-known for its superior biomass-assimilating ability, was also engineered to produce CA by expressing the $S$. maritimus PAL, which resulted in $210,450,460$, 300 and $130 \mathrm{mg} / \mathrm{L}$ of CA in shake-flask cultures from glucose, glycerol, raw starch, xylose and xylan, respectively [100]. By using $30 \mathrm{~g} / \mathrm{L}$ of cello- and xylo-oligosaccharide as carbon sources, CA production levels by engineered S. lividans reached 490 and $450 \mathrm{mg} / \mathrm{L}$, respectively [101]. The engineered $E$. coli strain expressing the PAL/TAL from Rhodotorula glutinis or A. thaliana was evaluated for CA production in different carbon sources (i.e., glucose, xylose or arabinose, as well as a mixture of these three sugars mimicking the lignocellulosic hydrolysate). As a result, $E$. coli carrying the A. thaliana TAL/PAL using arabinose produced the highest CA titer [102]. In addition to the conventional approach using TAL/ PAL, microbial CA production has also been achieved by the $f l d A B C I$-encoded phenyllactate dehydratase from Clostridium sporogenes [103-105]. Under optimized oxygen conditions, two-step bioprocess approach enabled $1.7 \mathrm{~g} / \mathrm{L}$ of CA through stepwise production of D-PhLA and $C A$ in $E$. coli strain expressing W. fluorescens pprA and $C$. sporogenes fldABCI, respectively [105]. In a most recent study, the highest reported CA titer of $6.9 \mathrm{~g} / \mathrm{L}$ was achieved by engineered $E$. coli harboring a PAL in a fedbatch culture [106].

Styrene is a petroleum-derived chemical produced from dehydrogenation of ethylbenzene, and it is also formed by non-oxidative decarboxylation of $\mathrm{CA}$ via microbial biosynthesis [107]. Although the bioconversion of CA to styrene was shown almost a century ago [108], the first artificial biosynthetic pathway for styrene production from glucose was only established recently, through deamination of L-PHE by the A. thaliana PAL2encoded lyase followed by decarboxylation of CA to styrene by the $S$. cerevisiae FDC1-encoded ferulate decarboxylase. While phenylacrylate decarboxylase from $S$. cerevisiae was also identified to be able to decarboxylate $\mathrm{CA}$, the higher styrene titer of $260 \mathrm{mg} / \mathrm{L}$ was achieved through the use of PAL2 and FDC1 [107]. A co-culture system of $S$. lividans strains expressing the $S$. cerevisiae FDC1 and $S$. maritimus PAL was developed to produce styrene from different carbon sources such as glucose, 


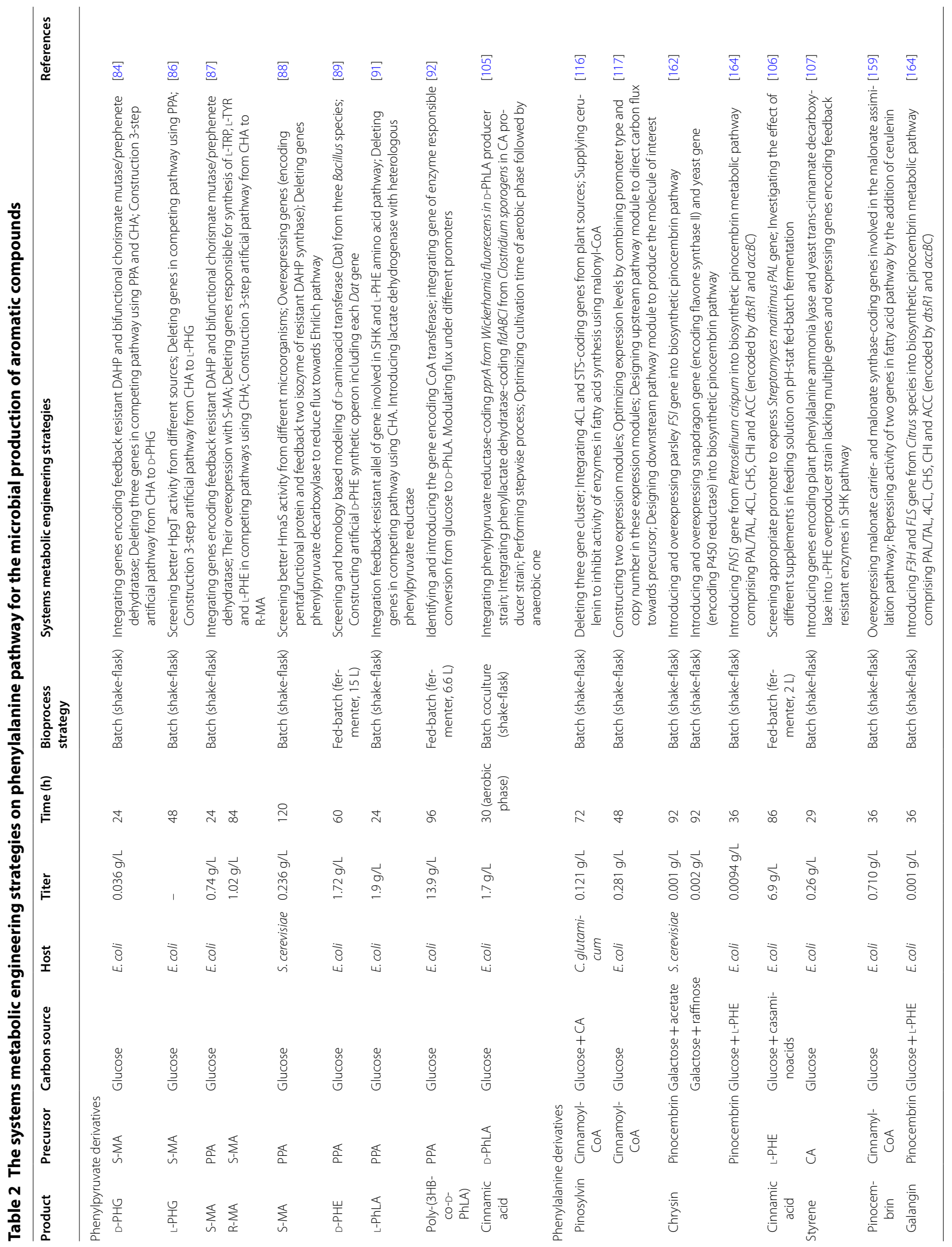




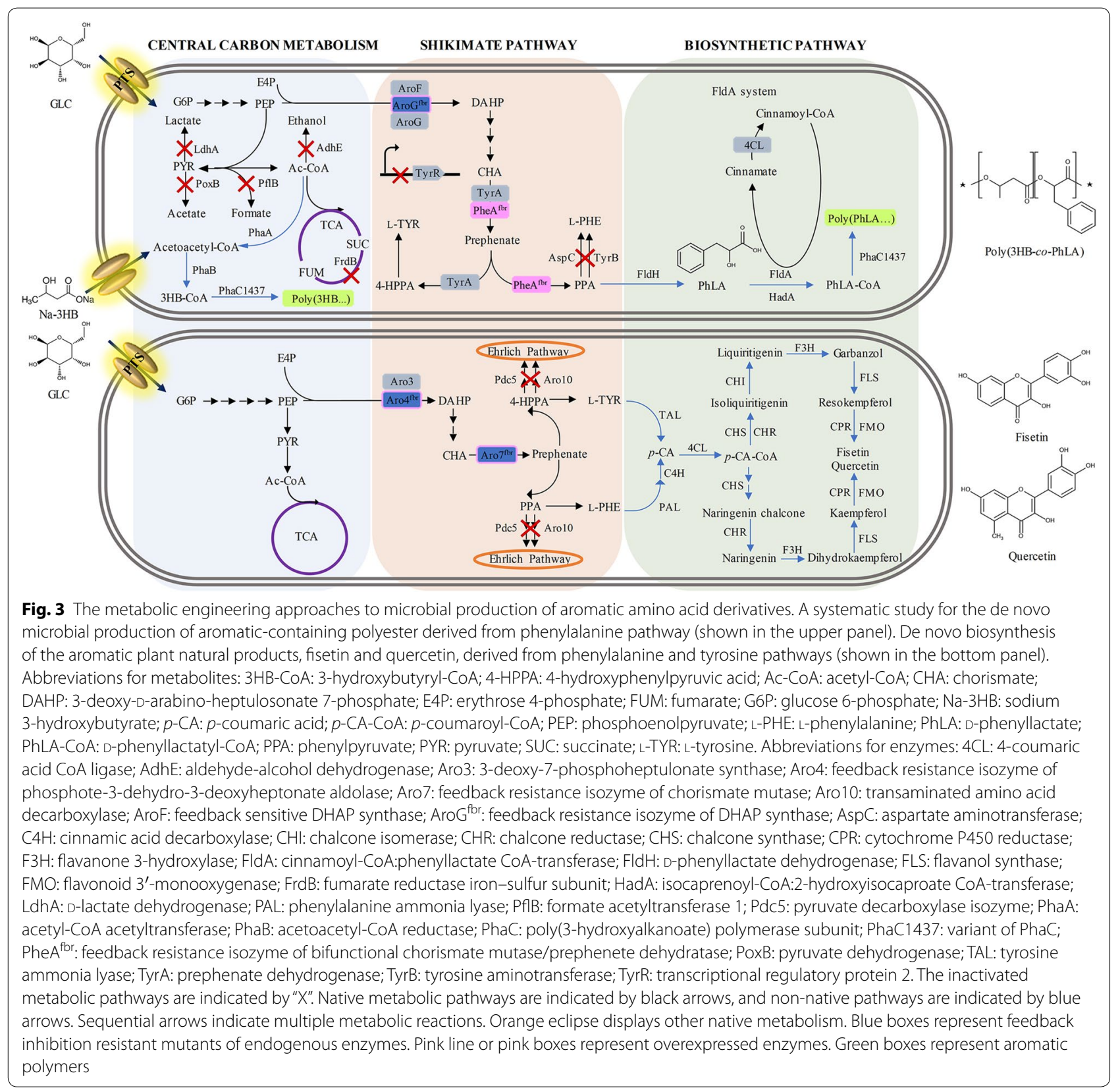

cellobiose or xylo-oligosaccharide, which however did not lead to an increase in styrene production [109].

Hydroxycinnamic acids are a group of CA derivatives, which mainly consist of $p$-hydroxycinnamic acid ( $p$-coumaric acid), 3,4-dihydroxycinnamic acid (caffeic acid) and 4-hydroxy-3-methoxycinnamic acid (ferulic acid) [110]. There is a sequential bioconversion from $p$-coumaric acid to ferulic acid through caffeic acid. These hydroxycinnamic acids will be described in the following section as they are derived from the L-TYR biosynthesis pathway.
Among various flavonoids, pinocembrin as a plant secondary metabolite is an important substance used in food and pharmaceutical industries thanks to its antiinflammatory, anti-microbial, anti-cancer and neuroprotective activities [111]. In plant metabolism, pinocembrin is a branch point for the biosynthesis of several other flavonoids including galangin, dihydroflavonol and chrysin, through enzymatic modifications in its core structure. Microbial production of pinocembrin and related flavonoids will be discussed later as both L-PHE and L-TYR 
biosynthesis pathways are involved in producing these molecules.

Among non-flavonoid phytochemicals in plants, stilbenes (stilbenoids) are polyphenolic secondary metabolites, which come to the fore with the 1,2-diphenylethylene nucleus. Similar to flavonoids, stilbenes are synthesized to defense plants against various environmental stresses such as wounding, fungal infection, UV radiation, etc. They are also important drug substances because of their anti-cancer, anti-inflammatory and anti-obesity activities [112, 113]. Well-known stilbenes produced by microbial systems are resveratrol, pterostilbene, piceatannol, pinosylvin, etc. The natural biosynthesis of stilbenes follows a similar pathway to those of flavonoids and phenylpropanoids through the intermediary formation of CoA esters, i.e., cinnamoyl-CoA and $p$-coumaroyl-CoA. These aromatic CoA esters, cinnamoyl-CoA and $p$-coumaroyl-CoA, are condensed with three malonyl-CoA units by stilbene synthase (STS) to produce the stilbene backbone, followed by the formation of pinosylvin and resveratrol, respectively. In a similar fashion, t-piceatannol is also produced using caffeic acid as a starter unit. Additionally, various more complex stilbenes are synthesized through modifications of the stilbene backbone using reactions including glycosylation, methylation, oligomerization and prenylation [114]. Microbial production of pinosylvin from glucose was accomplished in an engineered E. coli harboring PAL, 4-coumaric acid CoA-ligase (4CL) and STS. Through the use of an evolved STS with higher catalytic activity as well as the addition of cerulenin for increased malonylCoA pool and L-PHE for precursor supply, the pinosylvin titer was increased up to $91 \mathrm{mg} / \mathrm{L}$ [115]. Engineered $C$. glutamicum expressing the same set of enzymes responsible for pinosylvin synthesis was also developed, which produced $121 \mathrm{mg} / \mathrm{L}$ pinosylvin from CA supplemented [116]. Moreover, the highest reported pinosylvin titer of $281 \mathrm{mg} / \mathrm{L}$ was achieved by an engineered $E$. coli harboring the pinosylvin synthetic pathway optimized through a rational modular approach [117].

\section{L-TYR pathway derivatives}

The L-TYR biosynthesis pathway starts with the formation of 4-hydroxyphenylpyruvic acid (4-HPPA) from SHK pathway. Thus, microbial production of 4-HPPA and LTYR derivatives are dependent on this pathway (Table 3). Despite the biosynthesis of many L-TYR derivatives, only a few 4-HPPA derivatives have been biosynthesized in engineered microbes.

4-Hydroxyphenyllactic acid (4-HPLA) is known for its anti-fungal activity against several species of filamentous fungi, and it is naturally produced by Bifidobacteria and
Lactobacilli. The biosynthesized 4-HPLA via L-TYR in engineered microbes were used as a precursor to further produce other compounds such as aromatic polyesters [92] and salvianic acid A [118].

Salvianic acid A (also known as danshensu and 3 -( $3^{\prime}, 4^{\prime}$-dihydroxyphenyl)-2-hydroxypropanoic acid) is a natural compound in the plant Salvia miltiorrhiza, and it is attractive because of its anti-oxidant and therapeutic activities. Even though the natural biosynthesis pathway of salvianic acid A has not been fully revealed, an artificial biosynthetic pathway for salvianic acid A has been constructed in E. coli through a two-step bioconversion from 4-HPPA catalyzed by Lactobacillus pentosus D-lactate dehydrogenase (encoded by mutated $d$-ldh) and E. coli hpaBC-encoded hydroxylase complex [118]. Coexpression of these two enzymes resulted in two different metabolic pathways characterized by particular intermediates 4-HPLA and 3,4-dihydroxyphenylpyruvate, due to the different sequence of the reactions catalyzed (Fig. 1). To enhance flux towards 4-HPPA, a modular approach was applied by constructing three expression plasmids harboring the pathway genes within modules such as $\operatorname{aro} G^{\mathrm{fbr}}$-tyr $A^{\mathrm{fbr}}$-aroE, ppsA-tktA-glk and hpaBC-d-ld$h^{Y 52 A}$, along with deletion of $p t s G$, $p y k A$, pheA and tyrR. As a result, the highest reported salvianic acid A titer of $7.1 \mathrm{~g} / \mathrm{L}$ was achieved in shake-flask cultures [118]. Furthermore, these gene expression modules were integrated into the $E$. coli chromosome to overcome the issues with plasmid instability and requirement of expensive antibiotics, which however resulted in lower salvianic acid A titer [119].

Rosmarinic acid (RA) is an ester of 3,4-dihydroxyphenyllactic acid and caffeic acid, and is biosynthesized from L-PHE and L-TYR in several plants such as Rosmarinus officinalis, Salvia officinalis and Perilla frutescens [120]. RA and its derivatives such as isorinic acid and lithospermic acid are promising compounds in pharmaceutical and nutraceutical industries owing to their anti-oxidant, anti-bacterial, anti-inflammatory, anti-allergic, neuroprotective and anti-viral activities [121]. According to the elucidated metabolic pathway in Coleus blumei, RA is synthesized from two intermediate precursors 4-coumaroyl-CoA and 4-hydroxyphenyllactic acid (4-HPLA) derived from L-PHE and L-TYR, respectively. To obtain 4-coumaroyl-CoA, L-PHE is catalyzed sequentially by PAL, cinnamic acid 4-hydroxylase $(\mathrm{C} 4 \mathrm{H})$ and $4 \mathrm{CL}$, while 4-HPLA is produced from L-TYR by tyrosine aminotransferase and hydroxyphenylpyruvate reductase (HPPR). Thereafter, 4-coumaroyl-4'-hydroxyphenyllactate is yielded from 4-coumaroyl-CoA and 4-HPLA by RA synthase (RAS). In the last step, the above intermediate is hydroxylated by a cytochrome $\mathrm{P} 450$ monooxygenase to synthesize RA [122, 123]. Different from this natural 


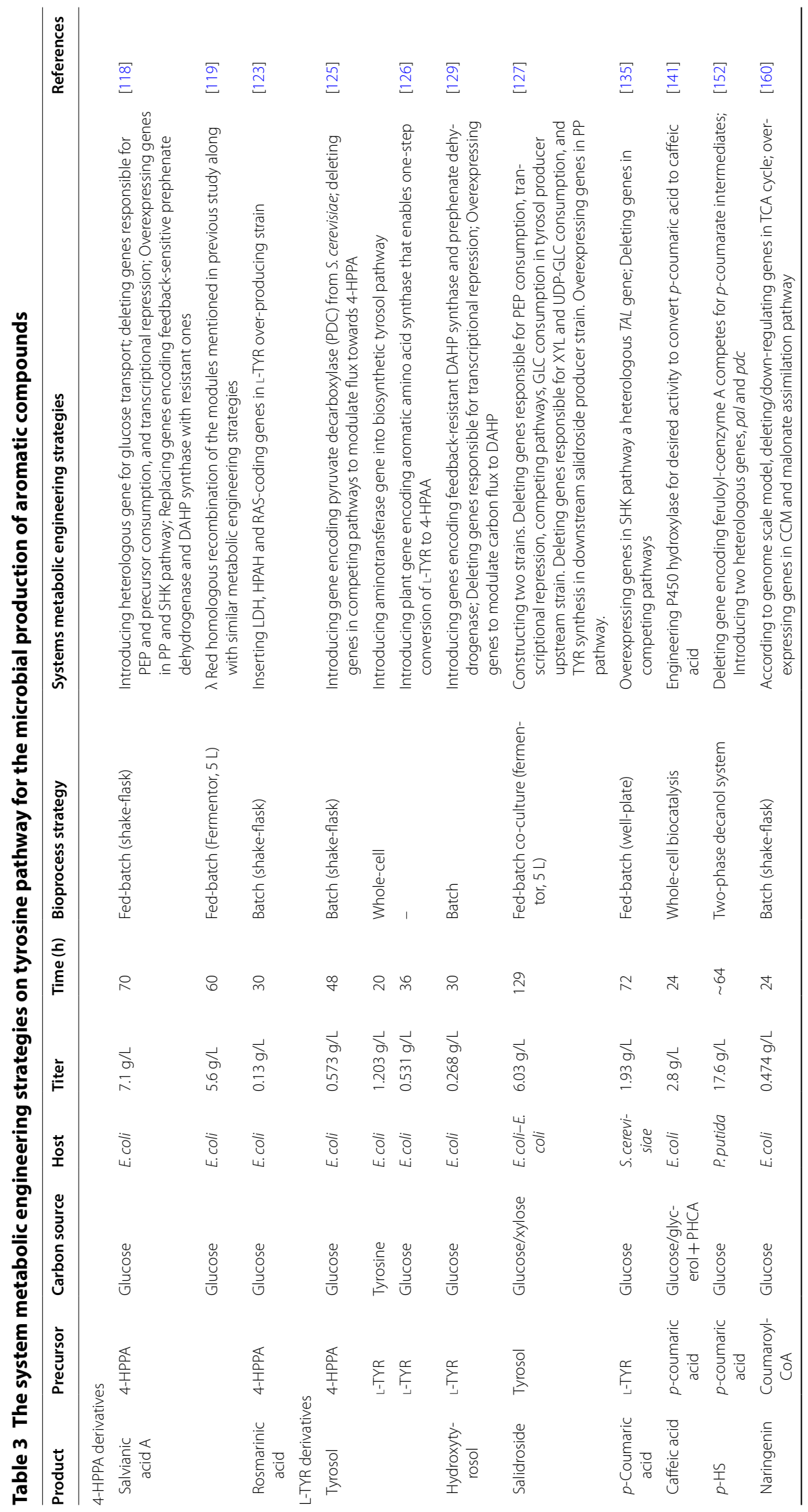




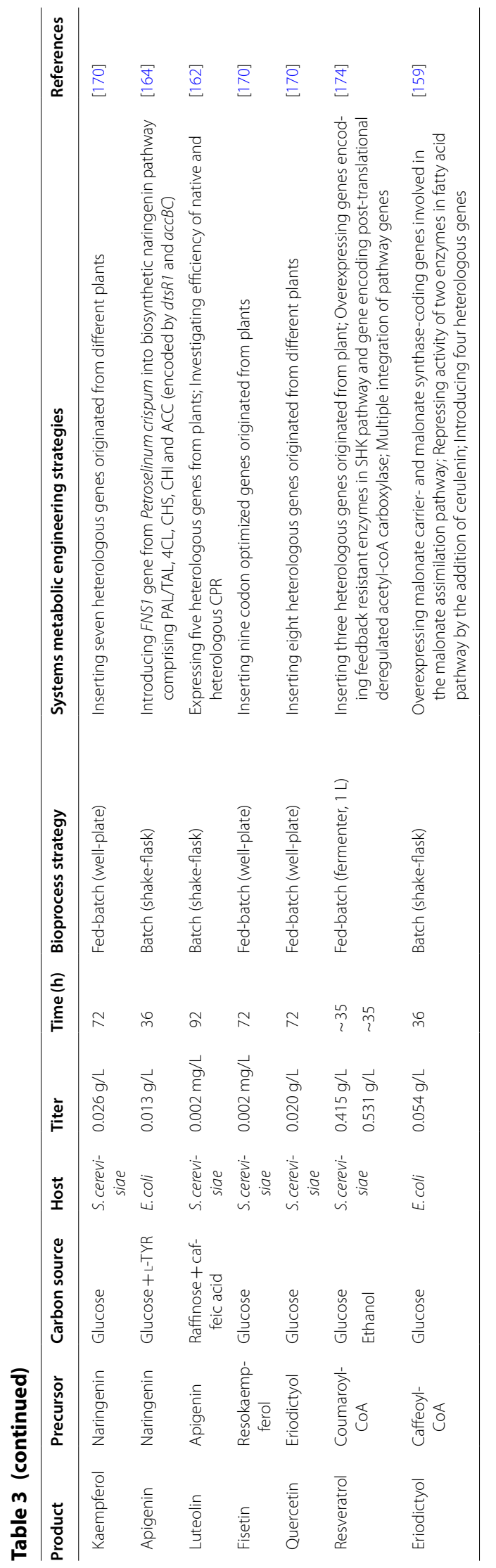


RA pathway, an artificial biosynthetic pathway was also constructed in a L-TYR-overproducing $E$. coli by expressing the A. thaliana 4CL, E. coli 4-hydroxyphenylacetate 3-hydroxylase (encoded by hpaBC), L. pentosus D-lactate dehydrogenase (encoded by $d-l d h^{\mathrm{Y} 52 \mathrm{~A}}$ ) and $C$. blumei RAS, as well as supplementing caffeic acid, which successfully produced 130 and $252 \mathrm{mg} / \mathrm{L}$ of RA and isorinic acid, as well as $55 \mathrm{mg} / \mathrm{L}$ of a novel compound caffeoylphenyllactate [123].

A group of phenolic compounds called phenylethanoids including tyrosol ( $p$-hydroxyphenylethanol), hydroxytyrosol and salidroside are derived from L-TYR pathway. Tyrosol and hydroxytyrosol are naturally present in olive oil and wine, and salidroside is found in the plant Rhodila rosea. These phenylethanoids are wellknown for their anti-oxidant activities, and they are industrially produced by chemical syntheses. The natural biosynthesis pathway for tyrosol via L-TYR in S. cerevisiae has been revealed and named as Ehrlich pathway. More specifically, L-TYR is sequentially converted to 4-HPPA, 4-hydroxyphenylacetaldehyde (4-HPAA) and tyrosol catalyzed by aminotransferase, 4-HPPA decarboxylase and alcohol dehydrogenase (ADH), respectively [124]. This yeast tyrosol pathway was established in an engineered $E$. coli strain with double deletion of pheA (encoding prephenate dehydratase) and $f e a B$ (encoding phenylacetaldehyde dehydrogenase). Also, the $S$. cerevisiae ARO10 and ARO8 genes encoding phenylpyruvate decarboxylase (PDC) and aminotransferase, respectively, were additionally expressed. Under optimized culture condition, the engineered $E$. coli expressing ARO10 produced $0.537 \mathrm{~g} / \mathrm{L}$ of tyrosol from glucose. By introducing ARO8 to this engineered E. coli, $1.203 \mathrm{~g} / \mathrm{L}$ of tyrosol was produced from $1.812 \mathrm{~g} / \mathrm{L}$ of L-TYR [125]. In a recent study, a recombinant $E$. coli expressing a gene encoding the aromatic acetaldehyde synthase (AAS) that is able to convert L-TYR to 4-HPAA in a single step was constructed. This engineered strain produced $531 \mathrm{mg} / \mathrm{L}$ tyrosol from glucose. Two more products could be produced by co-expressing additional genes in the above strain: $208 \mathrm{mg} / \mathrm{L}$ of hydroxytyrosol by co-expressing the hpaBC gene encoding $\mathrm{HpaBC}$ and $288 \mathrm{mg} / \mathrm{L}$ of salidroside by co-expressing a gene encoding uridine diphosphate-dependent glycosyltransferase (UGT) UGT85A1 [126]. Furthermore, E. coli-E. coli co-culture system was developed by separating biosynthetic pathways of tyrosol and salidroside, which led to production of $6.03 \mathrm{~g} / \mathrm{L}$ of salidroside from a glucose/xylose mixture [127].

An alternative tyrosol pathway was also established by expressing the genes encoding the L-TYR decarboxylase (TYDC) from Papaver somniferum, tyramine oxidase from Micrococcus luteus and E. coli endogenous ADHs in $E$. coli via the formation of tyramine and 4-HPAA.
Further deletion of $f e a B$ involved in the by-product 4-hydroxyphenylacetate formation increased tyrosol production to a titer of $68 \mathrm{mg} / \mathrm{L}$ from glucose [128]. Additional co-expression of $E$. coli 4-hydroxyphenylacetate 3-monooxygenase in an engineered $E$. coli expressing this synthetic tyrosol pathway led to production of $268.3 \mathrm{mg} / \mathrm{L}$ of hydroxytyrosol from glucose [129]. In addition, hydroxysalidroside was produced from hydroxytyrosol at a conversion yield of approximately $45 \%$ by the engineered E. coli expressing a selected UGT [129].

Microbial production of hydroxytyrosol was also achieved using an alternative biosynthetic pathway comprising the L-TYR hydroxylase (TH) from mouse, L-DOPA decarboxylase from pig and tyramine oxidase from $M$. luteus. In this strain, L-TYR was converted to hydroxytyrosol via L-3,4-dihydroxyphenylalanine (L-DOPA), dopamine and 3,4-dihydroxyphenylacetaldeyhde. This engineered strain was able to produce $0.08 \mathrm{mM}$ hydroxytyrosol from glucose. It was also reported that $E$. coli endogenous cofactor tetrahydromonapterin (MH4) could serve as an alternative cofactor to the eukaryotespecific cofactor tetrahydrobiophterin (BH4) required by $\mathrm{TH}[130]$.

$p$-Hydroxycinnamic acid (also known as $p$-coumaric acid), is synthesized by the $o$-hydroxylation of CA catalyzed by a CO-sensitive cytochrome $\mathrm{P} 450$ hydroxylase in plants [131]. Alternatively, $p$-coumaric acid is produced through the direct deamination of L-TYR by TAL in several microorganisms such as Rhodobacter capsulatus and Rhodobacter sphaeroides [132]. Enzymes responsible for this deamination reaction are classified into (1) TAL that solely converts L-TYR to $p$-coumaric acid and (2) PAL/ TAL that catalyzes not only the conversion of L-TYR to $p$-coumaric acid but also conversion of L-PHE to CA [97]. Due to the metabolic burden caused by the $\mathrm{P} 450$ hydroxylase, TAL-based approach is more desirable to synthesize $p$-coumaric acid in a microbial host. An engineered $P$. putida strain was constructed to produce $p$-coumaric acid from glucose via L-TYR. This engineered strain was further optimized through expression of a $m$-fluoro-DLphenylalanine-resistant mutant PAL, deletion of the feruloyl-CoA synthetase gene $(f c s)$ involved in $p$-coumaric acid degradation and construction of a L-PHE-auxotrophic mutant to avoid the accumulation of CA. Consequently, $1.7 \mathrm{~g} / \mathrm{L}$ of $p$-coumaric acid was produced from glucose in fed-batch culture [133]. Apart from pathway engineering, the choice of a TAL enzyme having higher activity and substrate specificity towards L-TYR is a key strategy to solve the bottleneck in the production of $p$-coumaric acid. To this end, TALs or PALs from diverse sources were investigated to identify the most efficient ones for $p$-coumaric acid synthesis in different hosts such as E. coli, Lactoccous lactis and S. cerevisiae. While 
the PALs from Brevibacillus laterosporus, Dictyostelium discoideum and Physcomitrella patens showed exclusive activity towards L-PHE, the TALs from Herpetosiphon aurantiacus and Flavobacterium johnsoniaeu exhibited higher reported substrate specificity towards L-TYR [134]. By expressing the $F$. johnsoniaeu TAL gene in an engineered $S$. cerevisiae strain lacking in genes encoding phenylpyruvate decarboxylase and pyruvate decarboxylase involved in competing pathways and overexpressing the genes encoding feedback-resistant DAHP synthase, CHA mutase and E. coli SHK kinase II, the highest reported $p$-coumaric acid titer of $1.93 \mathrm{~g} / \mathrm{L}$ was achieved from glucose in fed-batch culture [135].

3,4-Dihydroxycinnamic acid (caffeic acid) is a common phytochemical synthesized in plants together with ferulic acid. Caffeic acid and its phenethyl ester (CAPE) are used in the pharmaceutical and cosmetic industries due to their anti-oxidant, anti-aging and anti-carcinogenic activities. Despite its high demand on the market, caffeic acid is currently obtained by the extraction from plants [135] and its ester by the subsequent chemical synthesis [136]. In plants, caffeic acid is synthesized through three sequential reactions converting L-PHE to CA, $p$-coumaric acid, and finally to caffeic acid, which are catalyzed by PAL, cytochrome P450-dependent monooxygenase $(\mathrm{C} 4 \mathrm{H})$ and $p$-coumarate 3 -hydroxylase $(\mathrm{C} 3 \mathrm{H})$, respectively [137]. An alternative biosynthetic pathway of converting $\mathrm{L}$-TYR to caffeic acid via $p$-coumaric acid has also been identified in the actinomycete Saccharothrix espanaensis, which is composed of two reactions catalyzed by TAL (encoded by sam8) and C3H (encoded by sam5) [138]. The recombinant E. coli expressing the $S$. espanaensis sam 8 and sam5 genes successfully produced caffeic acid from glucose [139]. This two-step caffeic acid pathway has also been established by using alternative enzymes. The $E$. coli 4-hydroxyphenylacetate 3-hydroxylase encoded by the $h p a B C$ operon was shown to have a promiscuous activity of converting $p$-coumaric acid to caffeic acid. Co-expression of this hpaBC operon with the $R$. capsulatus TAL gene in a L-TYR-overproducing $E$. coli led to production of $50.2 \mathrm{mg} / \mathrm{L}$ of caffeic acid from glucose in shake flasks [140]. In addition, a bacterial P450 hydroxylase CYP199A2 from Rhodopseudomonas palustris that previously showed activity on carboxylic acids including 2-naphthoic acid, 4-ethylbenzoic acid, was engineered through site-directed mutagenesis to endow new capability to convert $p$-coumaric acid to caffeic acid. Using this engineered P450 hydroxylase, $2.8 \mathrm{~g} / \mathrm{L}$ of caffeic acid was produced through whole-cell biocatalysis [141].

In addition to the direct conversion of $p$-coumaric acid to caffeic acid by $\mathrm{C} 3 \mathrm{H}$ as described above, a threestep CoA-dependent pathway has also been identified to transform $p$-coumaric acid to caffeic acid [142-144].
In this novel pathway, $p$-coumaric acid is first converted to $p$-coumaroyl-CoA through the reaction catalyzed by $4 \mathrm{CL}$ using acetyl-CoA as a CoA donor [142]. Next, $p$-coumaroyl-CoA is hydroxylated to caffeoyl-CoA by $\mathrm{C} 3 \mathrm{H}$ [143]. Lastly, caffeoyl-CoA is hydrolyzed to form caffeic acid by CoA thioesterases [144]. An E. coli native hydroxyphenylacetyl-CoA thioesterase having broad substrate spectrum was also shown to act on caffeoyl-CoA to produce caffeic acid [145]. Based on these reaction steps, the CoA-dependent caffeic acid pathway was successfully established in the recombinant $E$. coli by expressing only the R. glutinis TAL, Petroselinum crispus 4CL and S. espanaensis $\mathrm{C} 3 \mathrm{H}$ genes [146].

4-Vinylphenol, also known as $p$-hydroxystyrene ( $p$ HS), is a versatile petroleum-derived platform chemical that can be used to produce a variety of polymers such as photoresist matrix polymer, resins and coatings. $p$-HS is also used to produce flavoring and fragrance substances in food, beverage and perfume industries [147]. $p$-HS and its derivatives 3,4-dihydroxystyrene and 4-hydroxy3-methoxystyrene are biosynthesized from $p$-coumaric acid, caffeic acid and ferulic acid, respectively. More specifically, $p$-HS is synthesized from L-TYR via $p$-coumaric acid through the two reactions catalyzed by TAL and phenolic acid decarboxylase (PAD). For 3,4-dihydroxystyrene synthesis, L-TYR is converted to $p$-coumaric acid, caffeic acid and finally to 3,4-dihydroxystyrene through the three reactions catalyzed by TAL, C3H and PAD, respectively. For 4-hydroxy-3-methoxystyrene synthesis, L-TYR is converted to $p$-coumaric acid, caffeic acid, ferulic acid and finally to 4-hydroxy-3-methoxystyrene through the four reactions catalyzed by TAL, C3H, caffeic acid methyltransferase and PAD, respectively. In the shake-flask culture of engineered E.coli strain expressing the S. espanaensis tal and sam5, Bacillus amyloliquefaciens PAD gene and $A$. thaliana caffeic acid methyltransferase gene, the titers of $p$-HS, 3,4-dihydroxystyrene and 4-hydroxy-3-methoxystyrene reached 355, 63 and $64 \mathrm{mg} / \mathrm{L}$ [148]. A variety of PADs from different microbial sources have been previously reported and it was shown that $p$-coumaric acid is the preferred substrate compared with caffeic acid and ferulic acid [149]. The engineered E. coli expressing the $R$. glutinis PAL gene and Lactobacillus plantarum PAD gene produced $0.4 \mathrm{~g} / \mathrm{L}$ of $p$-HS in glucose-fed batch fermentation. This $p$-HS titer was tenfold higher than that obtained with the E. coli strain expressing the Bacillus substilis PAD gene. In addition, the cytotoxicity of $p$-HS was observed via the decrease in the respiration curve and loss of $p$-coumaric acid decarboxylase activity [150]. Apart from the well-known microbial hosts, an engineered S. lividans expressing the $R$. sphaeroides TAL gene and Streptomyces sviceus PAD gene was shown to be prominent for $p$-HS production, 
as it could assimilate phosphoric acid swollen cellulose and convert it to $p$-HS at the yield of $94 \mathrm{~mol} \%$ without accumulation of the intermediate $p$-coumaric acid [151]. Due to the toxicity of $p$-HS to microbial cells, the solventtolerant $P$. putida S12 was also examined as a host to produce $p$-HS. The engineered $P$. putida strain was constructed by overexpressing the TAL and PAD genes and knocking out the $f c s$ gene encoding feruloyl-coenzyme A synthetase involved in competing pathway. In a twophase water/1-decanol fed-batch fermentation, this engineered $P$. putida strain produced $17.6 \mathrm{~g} / \mathrm{L}$ of $p$-HS [152].

Flavonoids are natural aromatic compounds produced in plants and fungi, and their skeleton is composed of benzopyrano moiety and benzene ring. According to the presence of additional rings and reduction/oxidation on pyrano ring, flavonoids are categorized into the subclasses of flavanols, flavanones and isoflavones. In natural biosynthesis of flavonoids, both L-PHE and L-TYR serve as precursors towards the formation of flavanone chalcones followed by formation of flavanones and flavonoids. At first, $p$-coumaric acid is produced either from L-PHE via CA through the reactions catalyzed by PAL and $\mathrm{C} 4 \mathrm{H}$ or from L-TYR catalyzed by TAL as described above. Then, $p$-coumaric acid is converted by $4 \mathrm{CL}$ to $p$-coumaroyl-CoA, which is reacted with three molecules of malonyl-CoA by chalcone synthase (CHS) to yield a C6-C3-C6 backbone unit "flavanone chalcone of naringenin". Subsequently, this chalcone is converted to flavanone by non-enzymatic reaction or chalcone isomerase $(\mathrm{CHI})$, and also serves as a precursor for the biosynthesis of other flavonoids [153].

Biosynthesis of the flavonoids pinocembrin and naringenin has been performed in engineered E. coli. On the basis of its broad substrate specificity, the $S$. coelicolor A3(2) 4CL, together with the Rhodotorula rubra PAL/TAL, was employed to simultaneously produce cinnamoyl-CoA and $p$-coumaroyl-CoA from L-PHE and L-TYR, respectively. These two CoA intermediates were further converted to the corresponding chalcones of pinocembrin and naringenin by $\mathrm{CHS}$ originating from the plant Glycyrrhiza echinata. The engineered E. coli harboring the above synthetic pathway produced 0.27 and $0.17 \mu \mathrm{g} / \mathrm{L}$ of naringenin and pinocembrin from glucose in shake-flask experiments, with the accumulation of 0.47 and $1.23 \mathrm{mg} / \mathrm{L}$ of $p$-coumaric acid and CA, respectively. When $2 \mathrm{mM}$ L-PHE and L-TYR were supplemented to increase precursor supply, 1.2- and 2-fold increase in the titers of pinocembrin and naringenin were observed [154]. When the genes encoding the plantoriginated $A$. thaliana $\mathrm{C} 4 \mathrm{H}$, Petroselinum crispum 4CL and Petunia $\times$ hybrida $\mathrm{CHI}$ and CHS were expressed in engineered $S$. cerevisiae using $\mathrm{CA}$ and $p$-coumaric acid as substrates, the titers of pinocembrin and naringenin reached 16.3 and $28.3 \mathrm{mg} / \mathrm{L}$, respectively. In another study, a recombinant $E$. coli was constructed by expressing the PAL, 4CL, CHS and $\mathrm{CHI}$ genes for pinocembrin synthesis. This recombinant strain was further engineered to enhance the malonyl-CoA pool by applying the CRISPR interference system. The resulting strain produced $525.8 \mathrm{mg} / \mathrm{L}$ of pinocembrin from glucose in a twostage $\mathrm{pH}$-controlled fed-batch culture [155]. Moreover, microbial production of eriodictyol was also achieved with a titer of $6.5 \mathrm{mg} / \mathrm{L}$ when caffeic acid was added [156].

In previous studies, the formation of $R$ - and $S$-form of flavonoids was not taken into consideration. However, it was reported that a racemic mixture of flavonoids was synthesized by the engineered $E$. coli with the lack of $\mathrm{CHI}$ activity. Also, it was identified that a bottleneck in the production of flavonoid was the limited pool of malonyl-CoA that was derived from acetyl-CoA by acetylCoA carboxylase (ACC). Engineered E. coli expressing the Pueraria lobate $\mathrm{CHI}$ and C. glutamicum ACC genes produced $1.01 \mathrm{mg} / \mathrm{L}$ of naringenin and $0.71 \mathrm{mg} / \mathrm{L}$ of pinocembrin in the presence of $2 \mathrm{mM} \mathrm{L}$-TYR and L-PHE, respectively. When the concentrated suspension of engineered cells was supplied with $3 \mathrm{mM} \mathrm{L}$-PHE and L-TYR, the pinocembrin and naringenin titers were increased to 58 and $57 \mathrm{mg} / \mathrm{L}$, respectively [157]. Co-expression of the Photorhabdus luminescens ACC gene in the engineered E. coli strain expressing the Petroselinum crispum 4CL, Petunia hybrida CHS and Medicago sativa CHI genes, the higher titers of pinocembrin, naringenin and eriodictyol reached 196, 67 and $17 \mathrm{mg} / \mathrm{L}$, respectively. Since ACC is a biotin-dependent carboxylase related to the biotin carboxyl carrier protein, the birA gene encoding a biotin-ligase was co-expressed with the ACC gene along with external addition of biotin. The resultant $E$. coli produced $367 \mathrm{mg} / \mathrm{L}$ of pinocembrin, $39 \mathrm{mg} / \mathrm{L}$ of naringenin and $50 \mathrm{mg} / \mathrm{L}$ of eriodictyol. By further overexpressing the $E$. coli acs gene encoding acetyl-CoA synthetase, the highest titers of pinocembrin, naringenin and eriodictyol of 429,119 and $52 \mathrm{mg} / \mathrm{L}$ were achieved, respectively. This was caused by the increase in cellular acetyl-CoA availability through acetate assimilation by the engineered E. coli strain. However, overexpression of the genes encoding acetate kinase and phosphate acetyltransferase resulted in slightly lower titers of these flavonoids compared with those obtained with the acs overexpression [158]. Modulation of the fluxes in other metabolic pathways such as malonate assimilation pathway and fatty acid pathway, was also shown to enhance production of pinocembrin, naringenin and eriodictyol [159].

Although flavonoids have been produced by engineered microbial systems as mentioned above, there is a drawback associated with the use of economically infeasible 
phenylpropanoic precursors. To replace these precursors with glucose, the pathway engineering strategies such as selection of appropriate enzyme candidates from different sources with codon-optimization and engineering malonyl-CoA pool, have been applied towards engineering of the four-step L-TYR-derived flavonoid biosynthetic pathway composed of TAL, 4CL, CHS and $\mathrm{CHI}$. By supplementing cerulenin as a fatty acid enzyme inhibitor, the naringenin titer of $84 \mathrm{mg} / \mathrm{L}$ was achieved by the engineered $E$. coli strain from glucose, almost threefold higher than that obtained without cerulenin [111]. Computational methods were also employed to aid the design of favorable strains producing higher amounts of desired products. For example, the application of genome-scale metabolic network modeling was reported to increase the production level of naringenin resulting from enhanced malonyl-CoA availability in engineered $E$. coli. Through deletion of the fumC (encoding fumarase) and sucC (encoding succinyl-CoA synthase) genes as well as overexpression of the genes encoding $\mathrm{ACC}$, phosphoglycerate kinase and pyruvate dehydrogenase, the engineered $E$. coli produced $474 \mathrm{mg} / \mathrm{L}$ of naringenin with the supplementation of $p$-coumaric acid [160]. When one or more gene interventions predicted by in silico flux analysis are carried out, an optimally balanced metabolic flux in the cell should be considered to avoid intermediate accumulation. To achieve balanced fluxes, the modular approach with the choice of appropriate gene copy number and promoter strength was also suggested [161].

The aforementioned artificial flavonoid pathway has also been expanded in microbial cell factories to produce various other aromatic natural products such as flavones (chrysin, apigenin, luteolin) and flavonols (kaempferol, quercetin, garbanzol, fisetin). By introduction of the soluble flavone synthase1 (FSI) from parsley into the aforementioned biosynthetic pathway established in $S$. cerevisiae [156], naringenin, pinocembrin and eriodictyol were converted to apigenin, chrysin and luteolin, respectively. By replacing FSI with the snapdragon insoluble flavone synthase II (FSII) (encoded by AFNS2) along with co-expression of the S. cerevisiae cytochrome P450 reductase (CPR1) gene, the apigenin titer was increased to 46 and $57 \mu \mathrm{g} / \mathrm{L}$ in raffinose- and acetate-containing media, respectively. A higher luteolin titer was achieved by using raffinose rather than acetate as carbon source. Chrysin production was eliminated due to the replacement of FSI with FSII [162]. To further produce methylated apigenin and luteolin, a recombinant $E$. coli strain was constructed by expressing the peppermint OMTIA gene (encoding 7-O-methyltransferase), parsley $4 C L$ and FSI genes, and the petunia $C H S$ and $C H I$ genes. The titers of apigenin and the methylated form of apigenin called genkwanin reached 415 and $208 \mu \mathrm{g} / \mathrm{L}$, respectively, when $p$-coumaric acid was fed in the presence of FSI activity. However, the luteolin titer was rather low and thus the methylated luteolin was not detected in the medium by using caffeic acid as carbon source [163]. By introducing the Citrus sinensis F3H (encoding flavanone 3-hydroxylase) and Citrus unshiu FLS (encoding flavanol synthase) genes into the engineered $E$. coli strain harboring the flavonoid biosynthetic pathway through expressing the genes encoding PAL/TAL, 4CL, CHS and CHI, $15.1 \mathrm{mg} / \mathrm{L}$ of kaempferol and $1.1 \mathrm{mg} / \mathrm{L}$ of galangin were achieved from L-TYR and L-PHE, respectively. Also, chrysin and apigenin were produced to concentrations of 9.4 and $13.1 \mathrm{mg} / \mathrm{L}$ from L-PHE and L-TYR, respectively, by using the $P$. crispum FSI [164]. Using a fusion protein of the Catharanthus roseus $3^{\prime}, 5^{\prime}$-hydroxylase $\left(\mathrm{F}^{\prime} 5^{\prime} \mathrm{H}\right)$ with CPR, the titers of kaempferol and quercetin were increased to 140 and $20 \mu \mathrm{g} / \mathrm{L}$, respectively, compared to those obtained without the chimeric enzyme. However, myricetin production was not detected [165]. Other chimeric enzymes including the Glycine max isofalavone synthase (ISFI) and $C$. roseus CPR were also employed in engineered $E$. coli and $S$. cerevisiae for the production of genistein and daidzein from naringenin and liquiritigenin, respectively. The product yield of genistein and daidzein in engineered bacteria reached 10 and $18 \mathrm{mg} / \mathrm{g} \mathrm{DCW}$, respectively, 20- and 9-fold higher than those obtained in engineered yeasts [166]. In another study, four engineered S. cerevisiae strains were constructed by expressing different combinations of genes encoding the following enzymes, PAL and CPR from Populus trichocarpa $\times P$. deltoides, $\mathrm{C} 4 \mathrm{H}, 4 \mathrm{CL}$, $\mathrm{CHS}, \mathrm{CHI}, \mathrm{F} 3 \mathrm{H}, \mathrm{F} 3^{\prime} \mathrm{H}$ and IFS from G. max, and FLS from Solanum tuberosu. These engineered yeast strains (named GEN23, KAE34, QUE44 and RESV11) enabled the production of genistein, kaempferol, quercetin and resveratrol from L-PHE [167]. The glucoside forms of isoflavonoids such as genistein $4{ }^{\prime}-O-\beta$-D-glucoside, genistin, genistein $4^{\prime}, 7-O-\beta$-D-diglucoside, sissotrin, daidzein $4^{\prime}, 7-O-\beta$-D-diglucoside, daidzein $4^{\prime}-O-\beta$-D-glucoside, daidzin, and ononin were also produced from respective isoflavonoid precursors catalyzed by the Bacillus licheniformis glycosyltransferase (encoded by $y j i C$ ) in engineered $E$. coli [168]. For fisetin synthesis, an artificial metabolic pathway was constructed, which was inspired from the quercetin pathway due to the structural similarity between them. Specifically, L-TYR is sequentially converted $p$-coumaric acid, $p$-coumaroyl-CoA, isoliquiritigenin, liquiritigenin, garbanzol, resokaempferol and finally to fisetin through the reactions catalyzed by TAL, 4CL, CHS-CHR, CHI, F3H, FLS and FMO/CPR, respectively. Engineered E. coli harboring this synthetic 
pathway produced $2.1 \mathrm{mg} / \mathrm{L}$ of fisetin with supplementation of resokaempferol [169]. Using a similar pathway approach, the engineered S. cerevisiae produced $20 \mathrm{mg} / \mathrm{L}$ of quercetin and $26 \mathrm{mg} / \mathrm{L}$ of kaempferol (Fig. 3) [170].

Microbial production of resveratrol has been achieved in engineered microbial systems based on the natural biosynthesis pathway of stilbenes. By introducing the hybrid poplar 4CL and Vitis vinifera resveratrol synthase genes into $S$. cerevisiae, the resveratrol was produced in the range of 100 to 300 nanogram per $200 \mathrm{~mL}$ culture [171]. The engineered $E$. coli strain expressing the $A$. thaliana 4CL and Arachis hypogaea STS genes produced up to $100 \mathrm{mg} / \mathrm{L}$ of resveratrol and $10 \mathrm{mg} / \mathrm{L}$ of piceatannol using glycerol as a carbon source with the supplementation of $p$-coumaric acid and caffeic acid, respectively. When glucose was used as carbon source, the resveratrol titer was decreased to $3.8 \mathrm{mg} / \mathrm{L}$, as glucose adversely affected the enzymatic activity and soluble expression of STS [172]. In another study, both engineered $E$. coli and $S$. cerevisiae were constructed for resveratrol production by expressing the plant-originated genes. In the engineered yeast, the Nicotiana tabacum 4CL gene was expressed under the control of GAL10 promoter while the Vitis vinifera STS gene was expressed under GAL1 promoter. The resultant yeast strain produced $6 \mathrm{mg} / \mathrm{L}$ of resveratrol. In the engineered $E$. coli, both of the genes were expressed under T7 promoter, which led to a higher resveratrol titer of $16 \mathrm{mg} / \mathrm{L}$ [173]. In another study using S. cerevisiae for resveratrol production, the engineered $S$. cerevisiae was first constructed by expressing the $H$. aurantiacus TAL, $A$. thaliana $4 \mathrm{CL}$ and $V$. vinifera STS genes. Then, the $\operatorname{aro}^{\mathrm{fbr}}$ (encoding a feedback-insensitive DAHP synthase), aro7 (encoding CHA mutase) and ACC1 (encoding a post-translational de-regulated acetyl-CoA carboxylase) genes were further overexpressed to enhance precursors supply of tyrosine and malonyl-CoA. This final strain produced 415.65 and $531.41 \mathrm{mg} / \mathrm{L}$ of resveratrol from glucose or ethanol in fed-batch fermentation, respectively [174]. A strategy of the facilitated transfer of cascade intermediates between 4CL and STS was applied by fusing the two enzymes together. For this purpose, the stop codon of $A$. thaliana $4 \mathrm{CL}$ gene was replaced with a linker sequence followed by the $V$. vinifera STS gene sequence. When this 4CL-STS fusion protein was expressed in S. cerevisiae, $5.25 \mu \mathrm{g} / \mathrm{mL}$ of resveratrol was produced, a 15 -fold increase than that obtained by the simple co-expression of these enzymes [175]. On the basis of resveratrol synthesis, various glycoside derivatives of resveratrol, such as resveratrol-4'-O-glucoside, resveratrol-3-O-glucoside and resveratrol-3-O-glucoside, have also been produced by engineered microbes by further expressing the $B$. licheniformis glycosyltransferase with broad substrate specificity against diverse nucleotide diphosphate sugars [176].

\section{L-TRP pathway derivatives}

In comparison to L-PHE and L-TYR pathways, L-TRP pathway has been relatively less studied, and thus has the greater potential to be exploited for biosynthesis of more and novel derivative aromatic compounds. A few L-TRP derivatives that have been produced by microbial systems are listed in Table 4.

Anthranilic acid is a metabolite of L-TRP biosynthesis pathway in microorganisms [177], and is also present in the carbazole degradation pathway of Pseudomonas stutzeri [178]. Currently, anthranilic acid is chemically synthesized from petroleum-derived $o$-xylene, and is mainly used in the synthesis of dyestuff, perfumes and pharmaceuticals [179]. To enable anthranilic acid accumulation by blocking the L-TRP pathway, a mutant $E$. coli strain having a nonsense mutation in the $\operatorname{trp} D$ gene (encoding the anthranilate phosphoribosyl transferase component of the anthranilate synthase-phosphoribosyl transferase complex) was generated by random mutagenesis. Overexpression of the genes that encode a feedback-resistant DAHP synthase and transketolase in this mutant $E$. coli resulted in production of $14 \mathrm{~g} / \mathrm{L}$ of anthranilic acid in fed-batch culture [180]. In another study, an engineered $P$. putida strain was also developed to produce anthranilic acid. In this engineered $P$. putida, the $\operatorname{trp} D C$ operon encoding anthranilate phosphoribosyltransferase (TrpD) and indole-3-glycerol phosphate synthase $(\operatorname{TrpC})$, and the pheA gene encoding chorismate mutase, were knocked out to eliminate competitive pathways. Further overexpression of the aro $G^{\text {D146N }}$ and $\operatorname{trp} E^{\mathrm{S} 40 \mathrm{~F}} G$ genes encoding the feedback-insensitive versions of DAHP synthase and anthranilate synthase, respectively, in this mutant $P$. putida strain led to production of $1.54 \mathrm{~g} / \mathrm{L}$ of anthranilic acid from glucose in a tryptophan-limited fed-batch culture [181].

Serotonin (5-hdyroxytryptamine) acts as a neurotransmitter in animals and also plays a role in regulating various physiological functions in plants. Serotonin is synthesized via L-TRP in both animals and plants but through different biosynthetic mechanisms. In animals, L-TRP is converted to serotonin via 5-hydroxytryptophan through the sequential reactions catalyzed by tryptophan 5-hydroxylase (TPH) and aromatic amino acid decarboxylase. Whereas in plants, L-TRP is first converted to tryptamine and then to serotonin by tryptophan decarboxylase (TDC) and tryptamine-5-hydoxylase (T-5H) [182]. This plant serotonin pathway was successfully established in a recombinant $E$. coli overexpressing the Catharanthus roseus TDC and rice T-5H genes. The soluble expression of $\mathrm{T}-5 \mathrm{H}$ was optimized by replacing the 


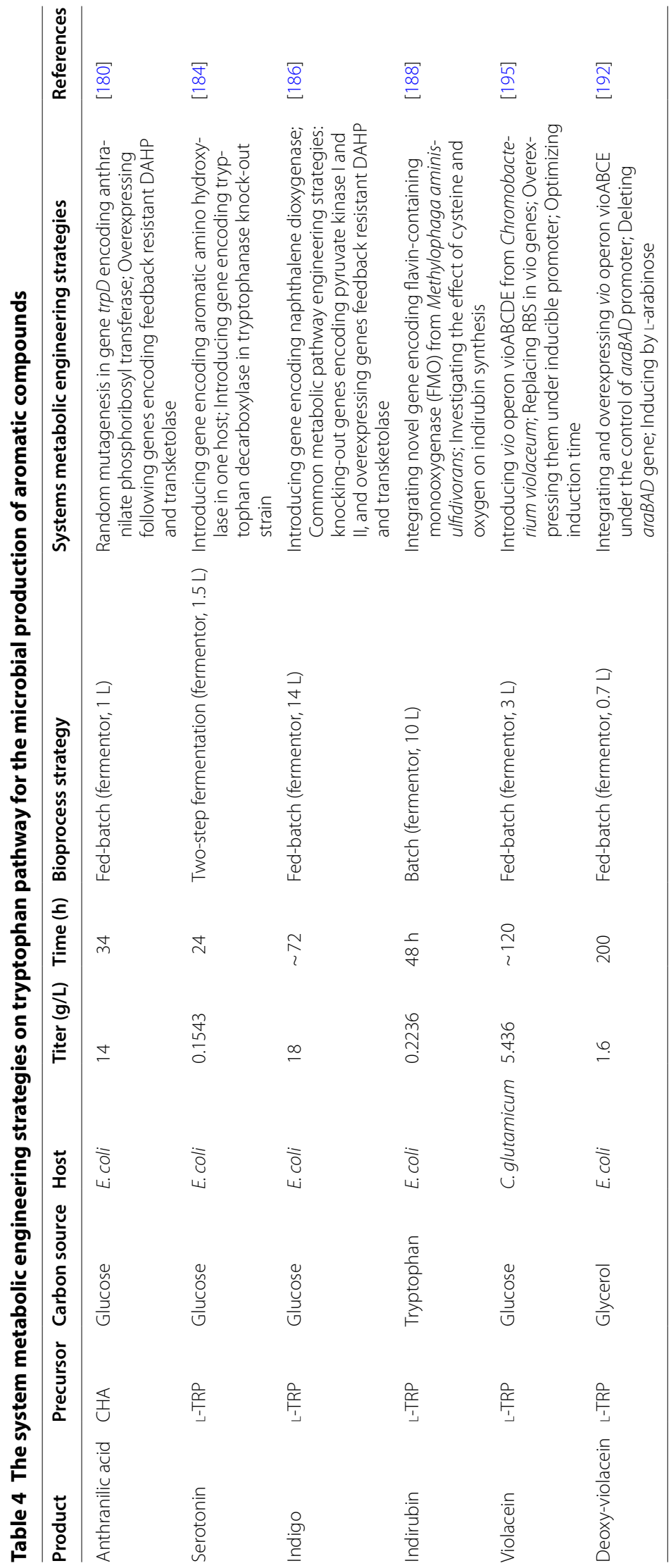


$\mathrm{N}$-terminal 37 amino acid residues with a glutathione $\mathrm{S}$ transferase (GST) tag. Under optimized condition, this engineered $E$. coli strain produced $24 \mathrm{mg} / \mathrm{L}$ of serotonin [183]. In a recent study, a stepwise system comprising two recombinant $E$. coli strains was constructed for serotonin production based on the animal serotonin pathway. In the first step, $962 \mathrm{mg} / \mathrm{L}$ of 5-hydroxytryptophan was produced from glucose by the recombinant $E$. coli strain expressing the gene encoding a mutated aromatic amino acid hydroxylase (CtAAAH) from Cupriavidus taiwanensis. Subsequently, bioconversion of the 5-hydroxytryptophan obtained in the first step led to production of $154.3 \mathrm{mg} / \mathrm{L}$ of serotonin by a second recombinant $E$. coli strain expressing the $C$. roseus TDC gene [184].

Indigo is a blue dye compound, which is obtained either by plant extraction or chemical synthesis. In some microorganisms such as Pseudomonas species, indigo is found to be produced from either indole or glucose as well as from degradation of particular aromatic compounds. Indigo and its isomer indirubin are synthesized from L-TRP by a three-step bioconversion: first, L-TRP is deaminated to indole by tryptophanase; then, indole is simultaneously oxidized to isatin, 2- and 3-hydroxyindole by monooxygenases or dioxygenases; lastly, spontaneous dimerization of these intermediates results in the formation of indigo and indirubin [185]. A recombinant $E$. coli strain expressing the $P$. putida naphthalene dioxygenase gene was constructed for indigo production. This $E$. coli was further engineered to enhance the L-TRP pathway flux by overexpressing the aro $G^{\mathrm{fbr}}$ and $t k t A$ genes encoding a feedback-resistant DAHP synthase and transketolase, respectively, and knocking out the $p y k F$ and $p y k A$ genes encoding pyruvate kinase I and II, respectively. The resultant engineered $E$. coli produced $18 \mathrm{~g} / \mathrm{L}$ of indigo from glucose in fed-batch culture [186]. On the other hand, the monooxygenase-mediated indigo production was also explored. A recombinant $E$. coli strain expressing the Methylophaga aminisulfidivorans $\mathrm{MP}^{\mathrm{T}}$ fmo gene encoding a flavin-containing monooxygenases (FMO) produced $920 \mathrm{mg} / \mathrm{L}$ of indigo from $2 \mathrm{~g} / \mathrm{L}$ of L-TRP in a batch culture [187].

Indirubin (also known as couraupitine B) is an indole alkaloid that serves as a drug substance used in the treatment of various diseases such as granulocytic leukemia, cancer and Alzheimer's disease. Indirubin is currently produced either by plant extraction or chemical synthesis. Production of indirubin has also been achieved in engineered microbial systems. In addition to indigo production, the aforementioned recombinant E. coli strain expressing the $f m o$ gene also produced $5.0 \mathrm{mg} / \mathrm{L}$ of indirubin from L-TRP. When this recombinant $E$. coli was cultured with supplementation of $0.36 \mathrm{~g} / \mathrm{L}$ of cysteine, a higher indirubin titer of $223.6 \mathrm{mg} / \mathrm{L}$ was achieved. It was speculated that the addition of cysteine enhanced synthesis of the indirubin precursor 2-hydroxyindole through affecting the regioselectivity of FMO [188]. Recently, microbial production of indirubin from glucose was accomplished by constructing an E. coli strain expressing the $M$. aminisulfidivorans fmo and $E$. coli tryptophanaseencoding tnaA genes. This recombinant $E$. coli strain was further engineered by knocking out $\operatorname{trp} R$ (encoding a regulatory repressor), $p y k F$ (encoding pyruvate kinase I) and $p y k A$ (encoding pyruvate kinase II), and overexpressing aro $G^{\mathrm{fbr}}$ (encoding a feedback-resistant DAHP synthase), $\operatorname{trp} E^{\mathrm{fbr}}$ (encoding a feedback-insensitive anthranilate synthase) and $t k t A$ (encoding transketolase). The resultant engineered $E$. coli strain successfully produced $56 \mathrm{mg} / \mathrm{L}$ of indirubin from glucose in fed-batch culture [189].

Violacein and deoxyviolacein are secondary metabolites found in microorganisms such as Collimona, Duganella, Janthinobacterium and Pseudoalteromonas, and are synthesized by condensation of two tryptophan-derived molecules [190]. Violacein and deoxyviolacein are considered as potential drug agents in pharmaceutical industry because of their anti-microbial, anti-tumor and anti-oxidant activities [186, 187]. Violacein is synthesized from L-TRP by the vioABCDE operon that encodes VioA, VioB, VioE, VioD and VioC, while deoxyviolacein synthesis requires the same set of enzymes except VioD [191]. A recombinant E. coli strain expressing the Chromobacterium violaceum vioABCE operon produced $180 \mathrm{mg} / \mathrm{L}$ of deoxyviolacein from glucose in shake flask cultures. Through systems-wide engineering of the serine, $\mathrm{CHA}$ and L-TRP biosynthesis and the non-oxidative PP pathway, bottlenecks in L-TRP supply were eliminated. As a result, a higher deoxyviolacein titer of $320 \mathrm{mg} / \mathrm{L}$ was obtained. Furthermore, co-expression of the Janthinobacterium lividum vioD gene in the above $E$. coli strain resulted in exclusive production of $710 \mathrm{mg} / \mathrm{L}$ of violacein from glucose in a fed-batch process [191]. In a following study, the $C$. violaceum vioABCE operon was overexpressed in a recombinant $E$. coli using the $\operatorname{araBAD}$ promoter induced by L-arabinose. In this $E$. coli strain, L-arabinose metabolism was also eliminated to prevent catabolism of the inducer L-arabinose by knocking out the ara $B A D$ genes. Using the resultant $E$. coli strain, $1.6 \mathrm{~g} / \mathrm{L}$ of deoxyviolacein was achieved in a glycerol-based fedbatch process [192]. Using another vioABCDE cluster from Duganella sp. B2, violacein production was investigated in three different bacterial hosts including $E$. coli, Citrobacter freundii and Enterobacter aerogenes. As a result, the highest violacein titer of $1.68 \mathrm{~g} / \mathrm{L}$ was achieved by the recombinant $C$. freundii strain expressing the Duganella sp. B2 vioABCDE operon in shake flask cultures [193]. When this recombinant $C$. freundii 
strain was cultured in a glycerol fed-batch process with optimized condition, $4.13 \mathrm{~g} / \mathrm{L}$ of violacein was obtained [194]. In another study, recombinant C. glutamicum strains were constructed by overexpressing the C. violaceum vioABCDE operon using different expression strategies, including the use of constitutive or inducible promoter, RBS replacement and reorganization of the vio operon. The best recombinant $C$. glutamicum strain produced $5.436 \mathrm{~g} / \mathrm{L}$ of violacein from glucose in an optimized fed-batch process [195].

Another interesting L-TRP derivative aromatic compound that has been recently studied is indole-3-acetic acid, also called auxin. Auxin is one of the phytohormones found in plants and is also synthesized in a range of phytobacteria such as Salmonella. A key enzyme for auxin synthesis called indole-3-pyruvate decarboxylase (IpdC) was identified in Salmonella. Heterologous expresssion of the Salmonella ipdC gene in the plant Medicago truncatula led to auxin production [196]. In the future, it is of great interest to explore auxin production in a recombinant microbial host expressing the Salmonella ipdC gene.

\section{Conclusions}

In this paper, we reviewed the current status of production of natural and non-natural aromatic chemicals by metabolically engineered microorganisms. Various metabolic engineering strategies and tools employed in the relevant works were reviewed. The aromatic chemicals that have been produced are classified according to their key precursors including SHK and aromatic amino acids. Some of the chemicals showcased in this paper are still produced at rather low efficiency, and thus need further strain improvement to enhance their production. It is expected that more and more aromatic compounds of industrial interest will be produced by microbial cell factories, either through the innovative rewiring of existing pathways or by identification of new enzymes and pathways. Complex regulatory circuits responsible for limited metabolic flux towards aromatic amino acids formation have been quite well known and thus do no longer present obstacle in increasing fluxes. For many aromatic products, in particular complex natural compounds, their production in engineered microorganisms is often limited by the activities of heterologous enzymes introduced for their production. Identification of better enzymes and also further improvement of these enzymes will play important roles in further enhancing production of various aromatic chemicals. As in the cases for developing strains overproducing other chemicals, systems metabolic engineering that integrates metabolic engineering with systems biology, synthetic biology and evolutionary engineering will offer more streamlined design of strategies for developing microbial strains efficiently producing various aromatic chemicals $[5,197]$.

\section{Authors' contributions}

All authors participated in the preparation of this contribution. All authors read and approved the final manuscript.

\section{Author details}

${ }^{1}$ Metabolic and Biomolecular Engineering National Research Laboratory, Department of Chemical and Biomolecular Engineering (BK21 Plus Program) and Institute for the BioCentury, Korea Advanced Institute of Science and Technology (KAIST), Daejeon 34141, Republic of Korea. ${ }^{2}$ Systems Metabolic Engineering and Systems Healthcare Cross-Generation Collaborative Laboratory, KAIST, Daejeon 34141, Republic of Korea. ${ }^{3}$ BioProcess Engineering Research Center and Bioinformatics Research Center, KAIST, Daejeon 34141, Republic of Korea.

\section{Acknowledgements}

Not applicable.

\section{Competing interests}

The authors declare that they have no competing interests.

\section{Availability of data and materials}

Data sharing not applicable to this article as no datasets were generated or analyzed during the current study.

Consent for publication

Not applicable.

Ethics approval and consent to participate

Not applicable.

\section{Funding}

The writing of the manuscript was supported by the Intelligent Synthetic Biology Center through the Global Frontier Project (2011-0031963) and also by the Technology Development Program to Solve Climate Changes on Systems Metabolic Engineering for Biorefineries (NRF-2012M1A2A2026556 and NRF-2012M1A2A2026557) from the Ministry of Science and ICT through the National Research Foundation of Korea.

\section{Publisher's Note}

Springer Nature remains neutral with regard to jurisdictional claims in published maps and institutional affiliations.

Received: 15 January 2019 Accepted: 19 February 2019

Published online: 26 February 2019

\section{References}

1. Noda S, Kondo A. Recent advances in microbial production of aromatic chemicals and derivatives. Trends Biotechnol. 2017;35:785-96.

2. Wu F, Cao P, Song G, Chen W, Wang Q. Expanding the repertoire of aromatic chemicals by microbial production. J Chem Technol Biotechnol. 2018;93:2804-16.

3. Bongaerts J, Krämer M, Müller U, Raeven L, Wubbolts M. Metabolic engineering for microbial production of aromatic amino acids and derived compounds. Metab Eng. 2001;3:289-300.

4. Cho C, Choi SY, Luo ZW, Lee SY. Recent advances in microbial production of fuels and chemicals using tools and strategies of systems metabolic engineering. Biotechnol Adv. 2015;33:1455-66.

5. Lee JW, Na D, Park JM, Lee J, Choi S, Lee SY. Systems metabolic engineering of microorganisms for natural and non-natural chemicals. Nat Chem Biol. 2012;8:536-46

6. Lee JW, Kim HU, Choi S, Yi J, Lee SY. Microbial production of building block chemicals and polymers. Curr Opin Biotechnol. 2011;22:758-67. 
7. Wang J, Shen X, Rey J, Yuan Q, Yan Y. Recent advances in microbial production of aromatic natural products and their derivatives. Appl Microbiol Biotechnol. 2018;102:47-61.

8. Deutscher J, Aké FMD, Derkaoui M, Zébré AC, Cao TN, Bouraoui H, et al. The bacterial phosphoenolpyruvate:carbohydrate phosphotransferase system: regulation by protein phosphorylation and phosphorylation-dependent protein-protein interactions. Microbiol Mol Biol Rev. 2014;78:231-56.

9. Li F-F, Zhao Y, Li B-Z, Qiao J-J, Zhao G-R. Engineering Escherichia coli for production of 4-hydroxymandelic acid using glucose-xylose mixture. Microb Cell Fact. 2016;15:90.

10. Floras N, Xiao J, Berry A, Bolivar F, Valle F. Pathway engineering for the production of aromatic compounds in Escherichia coli. Nat Biotechnol. 1996;14:620-3.

11. Flores S, Gosset G, Flores N, de Graaf AA, Bolivar F. Analysis of carbon metabolism in Escherichia coli strains with an inactive phosphotransferase system by ${ }^{13} \mathrm{C}$ labeling and NMR spectroscopy. Metab Eng. 2002:4:124-37.

12. Báez JL, Bolívar F, Gosset G. Determination of 3-deoxy-D-arabinoheptulosonate 7-phosphate productivity and yield from glucose in Escherichia coli devoid of the glucose phosphotransferase transport system. Biotechnol Bioeng. 2001;73:530-5.

13. Báez-Viveros J, Flores N, Juárez K, Castillo-España P, Bolivar F, Gosset G. Metabolic transcription analysis of engineered Escherichia coli strains that overproduce L-phenylalanine. Microb Cell Fact. 2007;6:30.

14. Yi J, Draths KM, Li K, Frost JW. Altered glucose transport and shikimate pathway product yields in Escherichia coli. Biotechnol Prog. 2003;19:1450-9.

15. Ikeda M, Mizuno Y, Awane S, Hayashi M, Mitsuhashi S, Takeno S. Identification and application of a different glucose uptake system that functions as an alternative to the phosphotransferase system in Corynebacterium glutamicum. Appl Microbiol Biotechnol. 2011;90:1443-51.

16. Shin W-S, Lee D, Lee SJ, Chun G-T, Choi S-S, Kim E-S, et al. Characterization of a non-phosphotransferase system for cis, cis-muconic acid production in Corynebacterium glutamicum. Biochem Biophys Res Commun. 2018;499:279-84.

17. Patnaik R, Liao JC. Engineering of Escherichia coli central metabolism for aromatic metabolite production with near theoretical yield. Appl Environ Microbiol. 1994:60:3903-8.

18. Draths KM, Frost JW. Synthesis using plasmid-based biocatalysis: plasmid assembly and 3-deoxy-D-arabino-heptulosonate production. J Am Chem Soc. 1990;112:1657-9.

19. Draths KM, Pompliano DL, Conley DL, Frost JW, Berry A, Disbrow GL, et al. Biocatalytic synthesis of aromatics from D-glucose: the role of transketolase. J Am Chem Soc. 1992;114:3956-62.

20. Nakahigashi K, Toya Y, Ishii N, Soga T, Hasegawa M, Watanabe H, et al. Systematic phenome analysis of Escherichia coli multiple-knockout mutants reveals hidden reactions in central carbon metabolism. Mol Syst Biol. 2009;5:1-14

21. Bentley R, Haslam E. The shikimate pathway - a metabolic tree with many branches. Crit Rev Biochem Mol Biol. 1990;25:307-84.

22. Herrmann KM, Weaver LM. The shikimate pathway. Annu Rev Plant Physiol Plant Mol Biol. 1999;50:473-503.

23. Jiang $\mathrm{M}$, Zhang $\mathrm{H}$. Engineering the shikimate pathway for biosynthesis of molecules with pharmaceutical activities in Escherichia coli. Curr Opin Biotechnol. 2016;42:1-6.

24. Herrmann KM. The shikimate pathway: early steps in the biosynthesis of aromatic compounds. Plant Cell. 1995;7:907-19.

25. Barker JL, Frost JW. Microbial synthesis of $p$-hydroxybenzoic acid from glucose. Biotechnol Bioeng. 2001;76:376-90.

26. Kitade $Y$, Hashimoto $R$, Suda M, Hiraga $K$, Inui M. Production of 4-hydroxybenzoic acid by an aerobic growth-arrested bioprocess using metabolically engineered Corynebacterium glutamicum. Appl Environ Microbiol. 2018;84:e02587-17.

27. Muller R, Wagener A, Schmidt K, Leistner E. Microbial production of specifically ring- ${ }^{13} \mathrm{C}$-labelled 4 -hydroxybenzoic acid. Appl Microbiol Biotechnol. 1995;43:985-8.

28. Krömer JO, Nunez-Bernal D, Averesch NJH, Hampe J, Varela J, Varela C. Production of aromatics in Saccharomyces cerevisiae-a feasibility study. J Biotechnol. 2013;163:184-93.
29. Williams TC, Averesch NJH, Winter G, Plan MR, Vickers CE, Nielsen LK, et al. Quorum-sensing linked RNA interference for dynamic metabolic pathway control in Saccharomyces cerevisiae. Metab Eng. 2015;29:124-34.

30. Zhang H, Pereira B, Li Z, Stephanopoulos G. Engineering Escherichia coli coculture systems for the production of biochemical products. Proc Natl Acad Sci. 2015;112:8266-71

31. van Duuren JBJH, Wijte D, Karge B, Martins dos Santos VAP, Yang Y, Mars $A E$, et al. $\mathrm{pH}$-stat fed-batch process to enhance the production of cis, cis-muconate from benzoate by Pseudomonas putida KT2440-JD1. Biotechnol Prog. 2012;28:85-92.

32. Sonoki T, Takahashi K, Sugita H, Hatamura M, Azuma Y, Sato T, et al. Glucose-free cis, cis-muconic acid production via new metabolic designs corresponding to the heterogeneity of lignin. ACS Sustain Chem Eng. 2018;6:1256-64.

33. Ornston $L N$. The conversion of catechol and protocatechuate to beta-ketoadipate by Pseudomonas putida. IV. Regulation. J Biol Chem. 1966;241:3800-10.

34. Fuchs $\mathrm{G}$, Boll M, Heider J. Microbial degradation of aromatic compounds — from one strategy to four. Nat Rev Microbiol. 2011;9:803-16.

35. Meganathan R. Ubiquinone biosynthesis in microorganisms. FEMS Microbiol Lett. 2001:203:131-9.

36. Averesch NJH, Krömer JO. Tailoring strain construction strategies for muconic acid production in Saccharomyces cerevisiae and Escherichia coli. Metab Eng Commun. 2014;1:19-28.

37. Draths KM, Frost JW. Environmentally compatible synthesis of adipic acid from D-glucose. J Am Chem Soc. 1994;116:399-400.

38. Niu W, Draths KM, Frost JW. Benzene-free synthesis of adipic acid. Biotechnol Prog. 2002;18:201-11.

39. Luo ZW, Kim WJ, Lee SY. Metabolic engineering of Escherichia coli for efficient production of 2-pyrone-4,6-dicarboxylic acid from glucose. ACS Synth Biol. 2018;7:2296-307.

40. Sun $X$, Lin $Y$, Huang Q, Yuan Q, Yan Y. A novel muconic acid biosynthesis approach by shunting tryptophan biosynthesis via anthranilate. Appl Environ Microbiol. 2013;79:4024-30

41. Sun X, Lin Y, Yuan Q, Yan Y. Biological production of muconic acid via a prokaryotic 2,3-dihydroxybenzoic acid decarboxylase. Chemsuschem. 2014:7:2478-81.

42. Sengupta S, Jonnalagadda S, Goonewardena L, Juturu V. Metabolic engineering of a novel muconic acid biosynthesis pathway via 4-hydroxybenzoic acid in Escherichia coli. Appl Environ Microbiol. 2015:81:8037-43.

43. Lin Y, Sun X, Yuan Q, Yan Y. Extending shikimate pathway for the production of muconic acid and its precursor salicylic acid in Escherichia coli. Metab Eng. 2014;23:62-9.

44. Weber C, Brückner C, Weinreb S, Lehr C, Essl C, Boles E. Biosynthesis of cis, cis-muconic acid and its aromatic precursors, catechol and protocatechuic acid, from renewable feedstocks by Saccharomyces cerevisiae. Appl Environ Microbiol. 2012;78:8421-30.

45. Curran KA, Leavitt JM, Karim AS, Alper HS. Metabolic engineering of muconic acid production in Saccharomyces cerevisiae. Metab Eng. 2013;15:55-66.

46. Johnson CW, Salvachúa D, Khanna P, Smith H, Peterson DJ, Beckham GT. Enhancing muconic acid production from glucose and lignin-derived aromatic compounds via increased protocatechuate decarboxylase activity. Metab Eng Commun. 2016;3:111-9.

47. Raj K, Partow S, Correia K, Khusnutdinova AN, Yakunin AF, Mahadevan R. Biocatalytic production of adipic acid from glucose using engineered Saccharomyces cerevisiae. Metab Eng Commun. 2018;6:28-32.

48. Converti A, Aliakbarian B, Domínguez JM, Vázquez GB, Perego P. Microbial production of biovanillin. Braz J Microbiol. 2010;41:519-30.

49. Gallage NJ, Møller BL. Vanillin-bioconversion and bioengineering of the most popular plant flavor and its de novo biosynthesis in the Vanilla Orchid. Mol Plant. 2015:8:40-57.

50. Sutherland JB, Crawford DL, Pometto AL. Metabolism of cinnamic, p-coumaric, and ferulic acids by Streptomyces setonii. Can J Microbiol. 1983;29:1253-7.

51. Achterholt S, Priefert $H$, Steinbüchel A. Identification of Amycolatopsis sp. strain HR167 genes, involved in the bioconversion of ferulic acid to vanillin. Appl Microbiol Biotechnol. 2000;54:799-807. 
52. Overhage J, Priefert H, Steinbüchel A. Biochemical and genetic analyses of ferulic acid catabolism in Pseudomonas sp. strain HR199. Appl Environ Microbiol. 1999;65:4837-47.

53. Plaggenborg $R$, Steinbüchel A, Priefert $H$. The coenzyme A-dependent, non- $\beta$-oxidation pathway and not direct deacetylation is the major route for ferulic acid degradation in Delftia acidovorans. FEMS Microbiol Lett. 2001;205:9-16.

54. Plaggenborg R, Overhage J, Steinbüchel A, Priefert H. Functional analyses of genes involved in the metabolism of ferulic acid in Pseudomonas putida KT2440. Appl Microbiol Biotechnol. 2003;61:528-35.

55. Di Gioia D, Luziatelli F, Negroni A, Ficca AG, Fava F, Ruzzi M. Metabolic engineering of Pseudomonas fluorescens for the production of vanillin from ferulic acid. J Biotechnol. 2011;156:309-16.

56. Lee EG, Yoon SH, Das A, Lee SH, Li C, Kim JY, et al. Directing vanillin production from ferulic acid by increased acetyl-CoA consumption in recombinant Escherichia coli. Biotechnol Bioeng. 2009;102:200-8.

57. Furuya T, Miura M, Kuroiwa M, Kino K. High-yield production of vanillin from ferulic acid by a coenzyme-independent decarboxylase/ oxygenase two-stage process. N Biotechnol. 2015;32:335-9.

58. Overhage J, Steinbüchel A, Priefert H. Highly efficient biotransformation of eugenol to ferulic acid and further conversion to vanillin in recombinant strains of Escherichia coli. Appl Environ Microbiol. 2003;69:6569-76.

59. Li K, Frost JW. Synthesis of vanillin from glucose. J Am Chem Soc. 1998;120:10545-6.

60. Hansen EH, Møller BL, Kock GR, Bünner CM, Kristensen C, Jensen OR et al. De novo biosynthesis of vanillin in fission yeast (Schizosaccharomyces pombe) and baker's yeast (Saccharomyces cerevisiae). Appl Environ Microbiol. 2009;75:2765-74.

61. Brochado A, Matos C, Møller BL, Hansen J, Mortensen UH, Patil $\mathrm{K}$. Improved vanillin production in baker's yeast through in silico design. Microb Cell Fact. 2010;9:84.

62. Furman BL. Salicylic acid. In: Reference Module in Biomedical Sciences 2018. doi:10.1016/B978-0-12-801238-3.97758-4.

63. Dempsey DA, Vlot AC, Wildermuth MC, Klessig DF. Salicylic acid biosynthesis and metabolism. Arabidopsis B. 2011;9:e0156.

64. Noda S, Shirai T, Oyama S, Kondo A. Metabolic design of a platform Escherichia coli strain producing various chorismate derivatives. Metab Eng. 2016;33:119-29.

65. Kallscheuer N, Marienhagen J. Corynebacterium glutamicum as platform for the production of hydroxybenzoic acids. Microb Cell Fact. 2018:17:70.

66. Qi R, Pfeifer BA, Zhang G. Engineering heterologous production of salicylate glucoside and glycosylated variants. Front Microbiol. 2018:9:1-7.

67. Ahmadi MK, Fang L, Moscatello N, Pfeifer BA. Escherichia coli metabolic engineering for gram scale production of a plant-based anti-inflammatory agent. Metab Eng. 2016;38:382-8.

68. Wegkamp A, van Oorschot W, de Vos WM, Smid EJ. Characterization of the role of $p$-aminobenzoic acid biosynthesis in folate production by Lactococcus lactis. Appl Environ Microbiol. 2007;73:2673-81.

69. Averesch NJH, Winter G, Krömer JO. Production of $p$-aminobenzoic acid from different carbon-sources in engineered Saccharomyces cerevisiae. Microb Cell Fact. 2016;15:89.

70. Koma D, Yamanaka H, Moriyoshi K, Sakai K, Masuda T, Sato Y, et al. Production of $p$-aminobenzoic acid by metabolically engineered Escherichia coli. Biosci Biotechnol Biochem. 2014;78:350-7.

71. Kubota T, Watanabe A, Suda M, Kogure T, Hiraga K, Inui M. Production of p-aminobenzoate by genetically engineered Corynebacterium glutamicum and non-biological formation of an N-glucosyl byproduct. Metab Eng. 2016;38:322-30.

72. Lekha PK, Lonsane BK. Production and application of tannin acyl hydrolase: state of the art. Adv Appl Microbiol. 1997;44:215-60.

73. Kambourakis S, Draths KM, Frost JW. Synthesis of gallic acid and pyrogallol from glucose: replacing natural product isolation with microbial catalysis. J Am Chem Soc. 2000;122:9042-3.

74. Wang J, Shen X, Yuan Q, Yan Y. Microbial synthesis of pyrogallol using genetically engineered Escherichia coli. Metab Eng. 2018;45:134-41.

75. Chen Z, Shen X, Wang J, Wang J, Yuan Q, Yan Y. Rational engineering of $p$-hydroxybenzoate hydroxylase to enable efficient gallic acid synthesis via a novel artificial biosynthetic pathway. Biotechnol Bioeng. 2017;114:2571-80.

76. Draths KM, Ward TL, Frost JW. Biocatalysis and nineteenth century organic chemistry: conversion of D-glucose into quinoid organics. J Am Chem Soc. 1992;114:9725-6.

77. Ran N, Knop DR, Draths KM, Frost JW. Benzene-free synthesis of hydroquinone. J Am Chem Soc. 2001;123:10927-34.

78. Seo D-H, Jung J-H, Lee J-E, Jeon E-J, Kim W, Park C-S. Biotechnological production of arbutins ( $a$ - and $\beta$-arbutins), skin-lightening agents, and their derivatives. Appl Microbiol Biotechnol. 2012;95:1417-25.

79. Shen X, Wang J, Wang J, Chen Z, Yuan Q, Yan Y. High-level de novo biosynthesis of arbutin in engineered Escherichia coli. Metab Eng. 2017:42:52-8.

80. Wang S, Fu C, Bilal M, Hu H, Wang W, Zhang X. Enhanced biosynthesis of arbutin by engineering shikimate pathway in Pseudomonas chlororaphis P3. Microb Cell Fact. 2018;17:174.

81. Zhu H, Dai P, Zhang W, Chen E, Han W, Chen C, et al. Enzymic synthesis of gastrodin through microbial transformation and purification of gastrodin biosynthesis enzyme. Biol Pharm Bull. 2010;33:1680-4.

82. Bai Y, Yin H, Bi H, Zhuang Y, Liu T, Ma Y. De novo biosynthesis of gastrodin in Escherichia coli. Metab Eng. 2016;35:138-47.

83. Masuo S, Zhou S, Kaneko T, Takaya N. Bacterial fermentation platform for producing artificial aromatic amines. Sci Rep. 2016;6:25764.

84. Müller U, van Assema F, Gunsior M, Orf S, Kremer S, Schipper D, et al. Metabolic engineering of the Escherichia coli L-phenylalanine pathway for the production of D-phenylglycine (D-Phg). Metab Eng. 2006;8:196-208.

85. Wegman MA, Janssen MHA, van Rantwijk F, Sheldon RA. Towards biocatalytic synthesis of $\beta$-lactam antibiotics. Adv Synth Catal. 2001;343:559-76.

86. Liu SP, Liu RX, El-Rotail AAMM, Ding ZY, Gu ZH, Zhang L, et al. Heterologous pathway for the production of L-phenylglycine from glucose by Escherichia coli. J Biotechnol. 2014;186:91-7.

87. Sun Z, Ning Y, Liu L, Liu Y, Sun B, Jiang W, et al. Metabolic engineering of the L-phenylalanine pathway in Escherichia coli for the production of S- or R-mandelic acid. Microb Cell Fact. 2011;10:71.

88. Reifenrath $M$, Boles E. Engineering of hydroxymandelate synthases and the aromatic amino acid pathway enables de novo biosynthesis of mandelic and 4-hydroxymandelic acid with Saccharomyces cerevisiae. Metab Eng. 2018;45:246-54.

89. Liu RX, Liu SP, Cheng S, Zhang L, Ding ZY, Gu ZH, et al. Screening, characterization and utilization of D-amino acid aminotransferase to obtain D-phenylalanine. Appl Biochem Microbiol. 2015;51:695-703.

90. Jamshidian M, Tehrany EA, Imran M, Jacquot M, Desobry S. Poly-lactic acid: production, applications, nanocomposites, and release studies. Compr Rev Food Sci Food Saf. 2010;9:552-71.

91. Fujita T, Nguyen HD, Ito T, Zhou S, Osada L, Tateyama S, et al. Microbial monomers custom-synthesized to build true bio-derived aromatic polymers. Appl Microbiol Biotechnol. 2013;97:8887-94.

92. Yang JE, Park SJ, Kim WJ, Kim HJ, Kim BJ, Lee H, et al. One-step fermentative production of aromatic polyesters from glucose by metabolically engineered Escherichia coli strains. Nat Commun. 2018;9:79.

93. Vogt T. Phenylpropanoid biosynthesis. Mol Plant. 2010;3:2-20.

94. Johnson JR. The perkin reaction and related reactions. Org React. 2011;210-265. https://doi.org/10.1002/0471264180.or001.08.

95. Jones G. The Knoevenagel condensation. Org React. 2011;204-599. https://doi.org/10.1002/0471264180.or015.02.

96. Koukol J, Conn EE. The metabolism of aromatic compounds in higher plants. IV. Purification and properties of the phenylalanine deaminase of Hordeum vulgare. J Biol Chem. 1961;236:2692-8.

97. Vannelli T, Wei Qi W, Sweigard J, Gatenby AA, Sariaslani FS. Production of $p$-hydroxycinnamic acid from glucose in Saccharomyces cerevisiae and Escherichia coli by expression of heterologous genes from plants and fungi. Metab Eng. 2007;9:142-51.

98. Xiang L. Inactivation, complementation, and heterologous expression of encP, a novel bacterial phenylalanine ammonia-lyase gene. J Biol Chem. 2002;277:32505-9.

99. Nijkamp K, van Luijk N, de Bont JAM, Wery J. The solvent-tolerant Pseudomonas putida $\mathrm{S} 12$ as host for the production of cinnamic acid from glucose. Appl Microbiol Biotechnol. 2005;69:170-7. 
100. Noda S, Miyazaki T, Miyoshi T, Miyake M, Okai N, Tanaka T, et al. Cinnamic acid production using Streptomyces lividans expressing phenylalanine ammonia lyase. J Ind Microbiol Biotechnol. 2011;38:643-8.

101. Noda S, Miyazaki T, Tanaka T, Ogino C, Kondo A. Production of Streptoverticillium cinnamoneum transglutaminase and cinnamic acid by recombinant Streptomyces lividans cultured on biomass-derived carbon sources. Bioresour Technol. 2012;104:648-51.

102. Vargas-Tah A, Martínez LM, Hernández-Chávez G, Rocha M, Martínez A, Bolívar $F$, et al. Production of cinnamic and $p$-hydroxycinnamic acid from sugar mixtures with engineered Escherichia coli. Microb Cell Fact. 2015;14:6.

103. Dickert S, Pierik AJ, Linder D, Buckel W. The involvement of coenzyme A esters in the dehydration of (R)-phenyllactate to (E)-cinnamate by Clostridium sporogenes. Eur J Biochem. 2000;267:3874-84.

104. Dickert S, Pierik AJ, Buckel W. Molecular characterization of phenyllactate dehydratase and its initiator from Clostridium sporogenes. Mol Microbiol. 2002;44:49-60.

105. Masuo S, Kobayashi Y, Oinuma K-I, Takaya N. Alternative fermentation pathway of cinnamic acid production via phenyllactic acid. Appl Microbiol Biotechnol. 2016:100:8701-9.

106. Bang HB, Lee K, Lee YJ, Jeong KJ. High-level production of trans-cinnamic acid by fed-batch cultivation of Escherichia coli. Process Biochem. 2018;68:30-6.

107. McKenna R, Nielsen DR. Styrene biosynthesis from glucose by engineered Escherichia coli. Metab Eng. 2011;13:544-54.

108. Arfmann H-A, Abraham W-R. Microbial formation of substituted styrenes. Zeitschrift für Naturforsch C. 1989:44:765-70.

109. Fujiwara R, Noda S, Tanaka T, Kondo A. Styrene production from a biomass-derived carbon source using a coculture system of phenylalanine ammonia lyase and phenylacrylic acid decarboxylase-expressing Streptomyces lividans transformants. J Biosci Bioeng. 2016:122:730-5.

110. Kim BG, Jung WD, Mok H, Ahn JH. Production of hydroxycinnamoylshikimates and chlorogenic acid in Escherichia coli: production of hydroxycinnamic acid conjugates. Microb Cell Fact. 2013;12:1-11.

111. Santos CNS, Koffas M, Stephanopoulos G. Optimization of a heterologous pathway for the production of flavonoids from glucose. Metab Eng. 2011;13:392-400.

112. Sirerol JA, Rodríguez ML, Mena S, Asensi MA, Estrela JM, Ortega AL. Role of natural stilbenes in the prevention of cancer. Oxid Med Cell Longev. 2016:2016:1-15.

113. Chou Y-C, Ho C-T, Pan M-H. Stilbenes: chemistry and molecular mechanisms of anti-obesity. Curr Pharmacol Rep. 2018:4:202-9.

114. Dubrovina AS, Kiselev KV. Regulation of stilbene biosynthesis in plants. Planta. 2017;246:597-623.

115. van Summeren-Wesenhagen PV, Marienhagen J. Metabolic engineering of Escherichia coli for the synthesis of the plant polyphenol pinosylvin. Appl Environ Microbiol. 2015:81:840-9.

116. Kallscheuer N, Vogt M, Stenzel A, Gätgens J, Bott M, Marienhagen J. Construction of a Corynebacterium glutamicum platform strain for the production of stilbenes and (2S)-flavanones. Metab Eng. 2016;38:47-55.

117. Wu J, Zhang X, Zhu Y, Tan Q, He J, Dong M. Rational modular design of metabolic network for efficient production of plant polyphenol pinosylvin. Sci Rep. 2017:7:1-15.

118. Yao Y-F, Wang C-S, Qiao J, Zhao G-R. Metabolic engineering of Escherichia coli for production of salvianic acid A via an artificial biosynthetic pathway. Metab Eng. 2013;19:79-87.

119. Zhou L, Ding Q, Jiang GZ, Liu ZN, Wang HY, Zhao GR. Chromosome engineering of Escherichia coli for constitutive production of salvianic acid A. Microb Cell Fact. 2017;16:1-11.

120. Kim GD, Park YS, Jin YH, Park CS. Production and applications of rosmarinic acid and structurally related compounds. Appl Microbiol Biotechnol. 2015;99:2083-92.

121. Petersen M, Simmonds MSJ. Rosmarinic acid. Phytochemistry 2003:62:121-5.

122. Petersen M. Rosmarinic acid: new aspects. Phytochem Rev. 2013;12:207-27.

123. Jiang J, Bi H, Zhuang Y, Liu S, Liu T, Ma Y. Engineered synthesis of rosmarinic acid in Escherichia coli resulting production of a new intermediate, caffeoyl-phenyllactate. Biotechnol Lett. 2016;38:81-8.

124. Hazelwood LA, Daran JM, Van Maris AJA, Pronk JT, Dickinson JR. The Ehrlich pathway for fusel alcohol production: a century of research on Saccharomyces cerevisiae metabolism. Appl Environ Microbiol. 2008;74:3920

125. Xue Y, Chen X, Yang C, Chang J, Shen W, Fan Y. Engineering Escherichia coli for enhanced tyrosol production. J Agric Food Chem. 2017:65:4708-14.

126. Chung D, Kim SY, Ahn JH. Production of three phenylethanoids, tyrosol, hydroxytyrosol, and salidroside, using plant genes expressing in Escherichia coli. Sci Rep. 2017;7:1-8.

127. Liu X, Li X-B, Jiang J, Liu Z-N, Qiao B, Li F-F, et al. Convergent engineering of syntrophic Escherichia coli coculture for efficient production of glycosides. Metab Eng. 2018;47:243-53.

128. Satoh Y, Tajima K, Munekata M, Keasling JD, Lee TS. Engineering of a tyrosol-producing pathway, utilizing simple sugar and the central metabolic tyrosine, in Escherichia coli. J Agric Food Chem. 2012;60:979-84.

129. Choo HJ, Kim EJ, Kim SY, Lee Y, Kim BG, Ahn JH. Microbial synthesis of hydroxytyrosol and hydroxysalidroside. Appl Biol Chem. 2018;61:295-301.

130. Satoh Y, Tajima K, Munekata M, Keasling JD, Lee TS. Engineering of L-tyrosine oxidation in Escherichia coli and microbial production of hydroxytyrosol. Metab Eng. 2012;14:603-10.

131. Gestetner B, Conn EE. The 2-hydroxylation of trans-cinnamic acid by chloroplasts from Melilotus alba desr. Arch Biochem Biophys. 1974;163:617-24

132. Kyndt JA, Meyer TE, Cusanovich MA, Van Beeumen JJ. Characterization of a bacterial tyrosine ammonia lyase, a biosynthetic enzyme for the photoactive yellow protein. FEBS Lett. 2002;512:240-4.

133. Nijkamp K, Westerhof RGM, Ballerstedt H, de Bont JAM, Wery J. Optimization of the solvent-tolerant Pseudomonas putida S12 as host for the production of $p$-coumarate from glucose. Appl Microbiol Biotechnol. 2007;74:617-24

134. Jendresen CB, Stahlhut SG, Li M, Gaspar P, Siedler S, Förster J, et al. Highly active and specific tyrosine ammonia-lyases from diverse origins enable enhanced production of aromatic compounds in bacteria and Saccharomyces cerevisiae. Appl Environ Microbiol. 2015;81:4458-76.

135. Rodriguez A, Kildegaard KR, Li M, Borodina I, Nielsen J. Establishment of a yeast platform strain for production of $p$-coumaric acid through metabolic engineering of aromatic amino acid biosynthesis. Metab Eng. 2015;31:181-8

136. Zhang P, Tang Y, Li N-G, Zhu Y, Duan J-A. Bioactivity and chemical synthesis of caffeic acid phenethyl ester and its derivatives. Molecules. 2014;19:16458-76.

137. Kojima M, Takeuchi W. Detection and characterization of $p$-coumaric acid hydroxylase in mung bean, Vigna mungo, seedlings. J Biochem. 1989;105:265-70.

138. Berner M, Krug D, Bihlmaier C, Vente A, Muller R, Bechthold A. Genes and enzymes involved in caffeic acid biosynthesis in the actinomycete Saccharothrix espanaensis. J Bacteriol. 2006;188:2666-73.

139. Choi O, Wu CZ, Kang SY, Ahn JS, Uhm TB, Hong YS. Biosynthesis of plant-specific phenylpropanoids by construction of an artificial biosynthetic pathway in Escherichia coli. J Ind Microbiol Biotechnol. 2011;38:1657-65.

140. Lin Y, Yan Y. Biosynthesis of caffeic acid in Escherichia coli using its endogenous hydroxylase complex. Microb Cell Fact. 2012;11:42.

141. Furuya T, Arai Y, Kino K. Biotechnological production of caffeic acid by bacterial cytochrome P450 CYP199A2. Appl Environ Microbiol. 2012;78:6087-94.

142. Gross GG, Zenk MH. Isolation and properties of hydroxycinnamate: CoA ligase from lignifying tissue of Forsythia. Eur J Biochem. 1974:42:453-9.

143. Kneusel RE, Matern U, Nicolay K. Formation of trans-caffeoyl-CoA from trans-4-coumaroyl-CoA by $\mathrm{Zn}^{2+}$-dependent enzymes in cultured plant cells and its activation by an elicitor-induced $\mathrm{pH}$ shift. Arch Biochem Biophys. 1989:269:455-62

144. del Carmen Ramirez-Ahumada M, Timmermann BN, Gang DR. Biosynthesis of curcuminoids and gingerols in turmeric (Curcuma longa) and ginger (Zingiber officinale): identification of curcuminoid synthase and hydroxycinnamoyl-CoA thioesterases. Phytochemistry. 2006;67:2017-29.

145. Zhuang Z, Song F, Zhao H, Li L, Cao J, Eisenstein E, et al. Divergence of function in the hot dog fold enzyme superfamily: the bacterial thioesterase YciA. Biochemistry. 2008;47:2789-96. 
146. Zhang H, Stephanopoulos G. Engineering Escherichia coli for caffeic acid biosynthesis from renewable sugars. Appl Microbiol Biotechnol. 2013;97:3333-41.

147. Bernini R, Mincione E, Barontini M, Provenzano G, Setti L. Obtaining 4-vinylphenols by decarboxylation of natural 4-hydroxycinnamic acids under microwave irradiation. Tetrahedron. 2007;63:9663-7.

148. Kang S-Y, Choi O, Lee JK, Ahn J-O, Ahn JS, Hwang BY, et al. Artificial de novo biosynthesis of hydroxystyrene derivatives in a tyrosine overproducing Escherichia coli strain. Microb Cell Fact. 2015;14:78.

149. Barthelmebs L, Divies C, Cavin J-F. Expression in Escherichia coli of native and chimeric phenolic acid decarboxylases with modified enzymatic activities and method for screening recombinant E. coli strains expressing these enzymes. Appl Environ Microbiol. 2001;67:1063-9.

150. Qi W, Vannelli T, Breinig S, Benbassat A, Gatenby A, Haynie S, et al. Functional expression of prokaryotic and eukaryotic genes in Escherichia coli for conversion of glucose to $p$-hydroxystyrene. Metab Eng. 2007:9:268-76

151. Noda S, Kawai Y, Tanaka T, Kondo A. 4-Vinylphenol biosynthesis from cellulose as the sole carbon source using phenolic acid decarboxylase- and tyrosine ammonia lyase-expressing Streptomyces lividans. Bioresour Technol. 2015;180:59-65.

152. Verhoef S, Wierckx N, Westerhof RGM, de Winde JH, Ruijssenaars HJ. Bioproduction of $p$-hydroxystyrene from glucose by the solventtolerant bacterium Pseudomonas putida S12 in a two-phase waterdecanol fermentation. Appl Environ Microbiol. 2009;75:931-6.

153. Pandey RP, Parajuli P, Koffas MAG, Sohng JK. Microbial production of natural and non-natural flavonoids: pathway engineering, directed evolution and systems/synthetic biology. Biotechnol Adv. 2016;34:634-62.

154. Hwang El, Kaneko M, Ohnishi Y, Horinouchi S. Production of plant-specific flavanones by Escherichia coli containing an artificial gene cluster. Appl Environ Microbiol. 2003;69:2699-706.

155. Wu J, Zhang X, Zhou J, Dong M. Efficient biosynthesis of (2S)pinocembrin from $\mathrm{D}$-glucose by integrating engineering central metabolic pathways with a pH-shift control strategy. Bioresour Technol. 2016;218:999-1007.

156. Yan Y, Kohli A, Koffas MAG. Biosynthesis of natural flavanones in Saccharomyces cerevisiae. Appl Environ Microbiol. 2005;71:5610-3.

157. Miyahisa I, Kaneko M, Funa N, Kawasaki H, Kojima H, Ohnishi Y, et al. Efficient production of (2S)-flavanones by Escherichia coli containing an artificial biosynthetic gene cluster. Appl Microbiol Biotechnol. 2005;68:498-504.

158. Leonard E, Lim K-H, Saw P-N, Koffas MAG. Engineering central metabolic pathways for high-level flavonoid production in Escherichia coli. Appl Environ Microbiol. 2007;73:3877-86.

159. Leonard E, Yan Y, Fowler ZL, Li Z, Lim C-G, Lim K-H, et al. Strain improvement of recombinant Escherichia coli for efficient production of plant flavonoids. Mol Pharm. 2008;5:257-65.

160. Xu P, Ranganathan S, Fowler ZL, Maranas CD, Koffas MAG. Genomescale metabolic network modeling results in minimal interventions that cooperatively force carbon flux towards malonyl-CoA. Metab Eng 2011;13:578-87.

161. Wu J, Zhou T, Du G, Zhou J, Chen J. Modular optimization of heterologous pathways for de novo synthesis of (2S)-naringenin in Escherichia coli. PLOS ONE. 2014;9:e101492.

162. Leonard E, Yan Y, Lim KH, Koffas MAG. Investigation of two distinct flavone synthases for plant-specific flavone biosynthesis in Saccharomyces cerevisiae. Appl Environ Microbiol. 2005;71:8241-8.

163. Leonard E, Chemler J, Kok HL, Koffas MAG. Expression of a soluble flavone synthase allows the biosynthesis of phytoestrogen derivatives in Escherichia coli. Appl Microbiol Biotechnol. 2006;70:85-91.

164. Miyahisa I, Funa N, Ohnishi Y, Martens S, Moriguchi T, Horinouchi S. Combinatorial biosynthesis of flavones and flavonols in Escherichia coli. Appl Microbiol Biotechnol. 2006;71:53-8.

165. Leonard E, Yan Y, Koffas MAG. Functional expression of a P450 flavonoid hydroxylase for the biosynthesis of plant-specific hydroxylated flavonols in Escherichia coli. Metab Eng. 2006;8:172-81.

166. Leonard E, Koffas MAG. Engineering of artificial plant cytochrome P450 enzymes for synthesis of isoflavones by Escherichia coli. Appl Environ Microbiol. 2007:73:7246-51.
167. Trantas E, Panopoulos N, Ververidis F. Metabolic engineering of the complete pathway leading to heterologous biosynthesis of various flavonoids and stilbenoids in Saccharomyces cerevisiae. Metab Eng. 2009;11:355-66.

168. Pandey RP, Parajuli P, Koirala N, Lee JH, Park YI, Sohng JK. Glucosylation of isoflavonoids in engineered Escherichia coli. Mol Cells. 2014;37:172-7.

169. Stahlhut SG, Siedler S, Malla S, Harrison SJ, Maury J, Neves AR, et al. Assembly of a novel biosynthetic pathway for production of the plant flavonoid fisetin in Escherichia coli. Metab Eng. 2015:31:84-93.

170. Rodriguez A, Strucko T, Stahlhut SG, Kristensen M, Svenssen DK, Forster $J$, et al. Metabolic engineering of yeast for fermentative production of flavonoids. Bioresour Technol. 2017;245:1645-54.

171. Becker J, Armstrong G, VanderMerwe M, Lambrechts M, Vivier M, Pretorius I. Metabolic engineering of for the synthesis of the wine-related antioxidant resveratrol. FEMS Yeast Res. 2003;4:79-85

172. Watts KT, Lee PC, Schmidt-Dannert C. Biosynthesis of plant-specific stilbene polyketides in metabolically engineered Escherichia coli. BMC Biotechnol. 2006:6:22

173. Beekwilder J, Wolswinkel R, Jonker $H$, Hall R, de Vos CHR, Bovy A. Production of resveratrol in recombinant microorganisms. Appl Environ Microbiol. 2006;72:5670-2.

174. Li M, Kildegaard KR, Chen Y, Rodriguez A, Borodina I, Nielsen J. De novo production of resveratrol from glucose or ethanol by engineered Saccharomyces cerevisiae. Metab Eng. 2015;32:1-11.

175. Zhang Y, Li S-Z, Li J, Pan X, Cahoon RE, Jaworski JG, et al. Using unnatural protein fusions to engineer resveratrol biosynthesis in yeast and mammalian cells. J Am Chem Soc. 2006:128:13030-1.

176. Pandey RP, Parajuli P, Shin JY, Lee J, Lee S, Hong YS, et al. Enzymatic biosynthesis of novel resveratrol glucoside and glycoside derivatives. Appl Environ Microbiol. 2014;80:7235-43.

177. Truffa-Bachi P, Cohen GN. Some aspects of amino acid biosynthesis in microorganisms. Annu Rev Biochem. 1968;37:79-108.

178. Larentis AL, Sampaio HCC, Carneiro CC, Martins OB, Alves TLM. Evaluation of growth, carbazole biodegradation and anthranilic acid production by Pseudomonas stutzeri. Braz J Chem Eng. 2011;28:37-44.

179. Wiklund P, Bergman J. The chemistry of anthranilic acid. Curr Org Synth 2006:3:379-402.

180. Balderas-Hernandez VE, Sabido-Ramos A, Silva P, Cabrera-Valladares N, Hernandez-Chavez G, Baez-Viveros JL, et al. Metabolic engineering for improving anthranilate synthesis from glucose in Escherichia coli. Microb Cell Fact. 2009;8:19.

181. Kuepper J, Dickler J, Biggel M, Behnken S, Jäger G, Wierckx N, et al. Metabolic engineering of Pseudomonas putida KT2440 to produce anthranilate from glucose. Front Microbiol. 2015;6:1-9.

182. Ramakrishna A, Giridhar P, Ravishankar GA. Phytoserotonin: a review. Plant Signal Behav. 2011:6:800-9.

183. Park S, Kang K, Lee SW, Ahn MJ, Bae JM, Back K. Production of serotonin by dual expression of tryptophan decarboxylase and tryptamine 5-hydroxylase in Escherichia coli. Appl Microbiol Biotechnol. 2011;89:1387-94.

184. Mora-Villalobos J-A, Zeng A-P. Synthetic pathways and processes for effective production of 5-hydroxytryptophan and serotonin from glucose in Escherichia coli. J Biol Eng. 2018;12:3.

185. Han X, Wang W, Xiao X. Microbial biosynthesis and biotransformation of indigo and indigo-like pigments. Chin J Biotechnol. 2008;24:921-6.

186. Berry A, Dodge T, Pepsin M, Weyler W. Application of metabolic engineering to improve both the production and use of biotech indigo. J Ind Microbiol Biotechnol. 2002:28:127-33.

187. Han GH, Shin HJ, Kim SW. Optimization of bio-indigo production by recombinant Escherichia coli harboring fmo gene. Enzyme Microb Technol. 2008;42:617-23.

188. Han GH, Gim GH, Kim W, Seo SI, Kim SW. Enhanced indirubin production in recombinant Escherichia coli harboring a flavin-containing monooxygenase gene by cysteine supplementation. J Biotechnol. 2012;164:179-87.

189. Du J, Yang D, Luo ZW, Lee SY. Metabolic engineering of Escherichia coli for the production of indirubin from glucose. J Biotechnol. 2018;267:19-28.

190. Choi SY, Yoon K-h, Lee Jl, Mitchell RJ. Violacein: properties and production of a versatile bacterial pigment. Biomed Res Int. 2015;2015:465056. https://doi.org/10.1155/2015/465056. 
191. Rodrigues AL, Trachtmann N, Becker J, Lohanatha AF, Blotenberg J, Bolten CJ, et al. Systems metabolic engineering of Escherichia coli for production of the antitumor drugs violacein and deoxyviolacein. Metab Eng. 2013;20:29-41.

192. Rodrigues AL, Becker J, de Souza Lima AO, Porto LM, Wittmann C. Systems metabolic engineering of Escherichia coli for gram scale production of the antitumor drug deoxyviolacein from glycerol. Biotechnol Bioeng. 2014;111:2280-9.

193. Jiang $P X$, Wang HS, Zhang C, Lou K, Xing XH. Reconstruction of the violacein biosynthetic pathway from Duganella sp. B2 in different heterologous hosts. Appl Microbiol Biotechnol. 2010;86:1077-88.

194. Yang C, Jiang P, Xiao S, Zhang C, Lou K, Xing XH. Fed-batch fermentation of recombinant Citrobacter freundii with expression of a violaceinsynthesizing gene cluster for efficient violacein production from glycerol. Biochem Eng J. 2011;57:55-62.
195. Sun H, Zhao D, Xiong B, Zhang C, Bi C. Engineering Corynebacterium glutamicum for violacein hyper production. Microb Cell Fact. 2016:15:148.

196. Cox CE, Brandl MT, de Moraes MH, Gunasekera S, Teplitski M. Production of the plant hormone auxin by Salmonella and its role in the interactions with plants and animals. Front Microbiol. 2018;8:1-10.

197. Lee SY, Kim HU, Chae TU, Cho JS, Kim JW, Shin JH, Kim DI, Ko YS, Jang WD, Jang Y-S. A comprehensive metabolic map for production of biobased chemicals. Nat Catal. 2019;2:18-33.
Ready to submit your research? Choose BMC and benefit from:

- fast, convenient online submission

- thorough peer review by experienced researchers in your field

- rapid publication on acceptance

- support for research data, including large and complex data types

- gold Open Access which fosters wider collaboration and increased citations

- maximum visibility for your research: over $100 \mathrm{M}$ website views per year

At BMC, research is always in progress.

Learn more biomedcentral.com/submissions 